\title{
HD 96446: a long-period binary with a strongly magnetic He-rich primary with $\beta$ Cephei pulsations ${ }^{\star}$
}

\author{
J. F. González ${ }^{1}$, M. Briquet ${ }^{2,3}$, N. Przybilla ${ }^{4}$, M.-F. Nieva ${ }^{4}$, P. De Cat ${ }^{5}$, S. Saesen $^{6}$, S. Hubrig ${ }^{2}$, A. Thoul ${ }^{3}$, \\ P. I. Pápics ${ }^{7}$, L. Palaversa ${ }^{6,8}$, D. Naef ${ }^{6}$, M. Neveu-Van Malle ${ }^{6}$, S. Järvinen ${ }^{2}$, K. R. Pollard ${ }^{9}$, P. Kilmartin ${ }^{9}$, \\ N. Mowlavi ${ }^{6}$, and K. Butler ${ }^{10}$ \\ 1 Instituto de Ciencias Astronómicas, de la Tierra y del Espacio (CONICET-Universidad Nacional de San Juan), \\ Casilla de Correo 49, 5400 San Juan, Argentina \\ e-mail: jfgonzalez@conicet.gov.ar \\ 2 Leibniz-Institut für Astrophysik Potsdam (AIP), An der Sternwarte 16, 14482 Potsdam, Germany \\ ${ }^{3}$ Space sciences, Technologies and Astrophysics Research (STAR) Institute, Université de Liège, Allée du 6 Août 19C, Bât. B5C, \\ 4000 Liège, Belgium \\ ${ }^{4}$ Institut für Astro- und Teilchenphysik, Universität Innsbruck, Technikerstr. 25/8, 6020 Innsbruck, Austria \\ 5 Royal Observatory of Belgium, Ringlaan 3, 1180 Brussels, Belgium \\ 6 Département d'astronomie de l'Université de Genève, Chemin des Maillettes 51, 1290 Versoix, Switzerland \\ 7 Instituut voor Sterrenkunde, KU Leuven, Celestijnenlaan 200D, 3001 Leuven, Belgium \\ 8 Institute of Astronomy, University of Cambridge, Madingley Road, Cambridge CB3 0HA, UK \\ 9 Department of Physics and Astronomy, University of Canterbury, Private Bag 4800, Christchurch, New Zealand \\ 10 Universitäts-Sternwarte München, Scheinerstr. 1, 81679 München, Germany
}

Received 31 January 2019 / Accepted 17 April 2019

\begin{abstract}
Aims. HD 96446 is a magnetic B2p He-strong star that has been reported to be a $\beta$ Cep pulsator. We present a detailed spectroscopic analysis of this object based on an intensive observational data set obtained in a multisite campaign with the spectrographs CORALIE, FEROS, and HARPS (La Silla); UVES (Paranal); HERCULES (Mt. John Observatory); and GIRAFFE (SAAO).

Methods. Radial velocities were measured by cross-correlations and analysed to detect periodic variations. On the other hand, the mean spectrum was fit with spectral synthesis to derive atmospheric parameters and chemical abundances.

Results. From the analysis of radial velocities, HD 96446 was found to be a spectroscopic binary with a period of 799 days. The stellar companion, which contributes only $\sim 5 \%$ of the total flux, is an A0-type star. A frequency analysis of the radial velocities allowed us to detect two pulsational modes with periods $2.23 \mathrm{~h}$ and $2.66 \mathrm{~h}$. The main mode is most probably a low-inclination, dipole mode $(l, m)=(1,0)$, and the second pulsation mode corresponds to $(l, m)=(2,2)$ or to a pole-on $(l, m)=(3,2)$ configuration. In addition to radial velocities, the main pulsation mode is evidenced through small variations in the spectral morphology (temperature variations) and the light flux. The rotation period of $23.4 \mathrm{~d}$, was detected through the variation in line intensities. Chemical abundances are unevenly distributed over the stellar surface, with helium concentrated at the negative magnetic pole and most metals strengthened at lower latitudes. The mean chemical abundance of helium is strongly abnormal, reaching a value of 0.60 (number fraction).
\end{abstract}

Key words. binaries: spectroscopic - stars: chemically peculiar - stars: early-type - stars: magnetic field - stars: oscillations stars: individual: HD 96446

\section{Introduction}

According to their observed properties, several classes of stars have been defined among the main-sequence B-type stars. The two main groups, which are not mutually exclusive, are chemically peculiar (CP) B stars called Bp stars and B stars that undergo stellar pulsations. The Bp stars show abnormal abundances of certain chemical elements in their atmosphere. Among them, the helium-strong stars (with typically abundance ratios of $\mathrm{He} / \mathrm{H} \sim 0.5)$ are the hottest $\mathrm{CP}$ stars in the temperature range of 20000-25000 K (e.g. Smith 1996). In this part of the upper main sequence, we also observe $\beta$ Cep stars and hybrid $\mathrm{B}$ pulsators with both $\beta$ Cep (low-order $p$ and $g$-modes) and slowly pulsating B (SPB) stars (high-order

\footnotetext{
* Table 3 (RV data) is only available at the CDS via anonymous ftp to cdsarc.u-strasbg. fr $(130.79 .128 .5)$ or via http://cdsarc. u-strasbg.fr/viz-bin/qcat?J/A+A/626/A94
}

$g$-modes) pulsations. Their pulsations are driven by the so-called $\kappa$-mechanism (Dziembowski \& Pamiatnykh 1993). Stochastic oscillations have also been detected in a few hot stars (e.g. Belkacem et al. 2009; Neiner et al. 2012a). The study of pulsators allows us to probe the internal properties and physical processes acting in their interiors (Aerts et al. 2010).

It is well known that He-strong stars are usually found to possess strong large-scaled organized magnetic fields (Borra \& Landstreet 1979); instead, chemical peculiarities are very frequently detected at the surface of magnetic hot stars. The first detection of a magnetic field in a pulsating B star was presented by Henrichs et al. (2000) for the star $\beta$ Cephei. Since then, magnetic fields have been detected in other $\beta$ Cep and SPB stars (e.g. Neiner et al. 2003; Hubrig et al. 2006). Magnetic Bp pulsators are particularly important as they allow us to study the effects of magnetism on the stellar surface and on the stellar interior. First, the magnetic field has an effect on atomic diffusion in 
the atmospheres of Bp stars (Michaud et al. 1981). It suppresses turbulence leading to vertical and horizontal chemical inhomogeneities. Second, magnetic fields deeply modify the transport of angular momentum and the mixing of chemicals in stellar interiors. Above a critical field limit, the fields enforce a uniform rotation along field lines and the mixing is inhibited in the radiative zone of these stars (Mathis \& Zahn 2005; Zahn 2011). Asteroseismology gives us a unique opportunity to probe the role of magnetism inside stars by means of the observed pulsation modes, as performed for the magnetic He-strong $\beta$ Cep star V2052 Oph (Neiner et al. 2012b; Briquet et al. 2012) and for the pulsating magnetic B3.5V star HD 43317 (Buysschaert et al. 2018).

Although important progress has been achieved in the area of stellar magnetism over the last two decades, the nature and origin of magnetic fields in stars with radiative envelopes are not yet fully understood. Magnetic fields could be fossil relics of the fields that were present in the interstellar medium from which the stars formed (e.g. Moss 2003). Different scenarios to explain why only a subclass of early-type stars are observably magnetic in the framework of the fossil field theory were discussed by Borra et al. (1982). This could also be the result of the merging of components of a close binary (Ferrario et al. 2009; Tutukov \& Fedorova 2010; Wickramasinghe et al. 2014). To test the proposed scenarios, further observational efforts are needed.

The star HD 96446 (=V430 Car) is a well-known magnetic B2p He-strong photometric variable (Mathys 1991, and references therein) with longitudinal magnetic field measurements of the order of $-1 \mathrm{kG}$, indicating a strong polar field strength. More recently, the star was discovered to be a $\beta$ Cep pulsator with a period of $2.23 \mathrm{~h}$ (Neiner et al. 2012a). Therefore, this target is one of the rare pulsating early B-type stars with a strong magnetic field. The first analyses of its magnetic field geometry were hampered by the absence of a reliable rotation period (Borra \& Landstreet 1979; Bohlender et al. 1987; Mathys 1991; Neiner et al. 2012a) and of a sophisticated procedure to account for the impact of pulsations on the magnetic field measurements.

Given the strong potential of HD 96446 to provide new insights into the physics and origin of magnetic fields in massive stars, we organized a magneto-asteroseismology study of it. Using archival and new high-resolution spectropolarimetric observations, a first reliable magnetic field model of the target was obtained (Järvinen et al. 2017) and a dipole field strength of $4.64 \pm 0.88 \mathrm{kG}$ was derived. We also set up a spectroscopic multisite campaign to gather an intensive data set of high $\mathrm{S} / \mathrm{N}$ high-resolution spectra for asteroseismic purposes. In this paper we present a detailed analysis of our spectroscopy and we provide a discussion of our findings. In Sects. 2 and 3 we describe the observations and the fitting of the radial velocity variations. In Sect. 4 we discuss physical and atmospheric parameters, and derive chemical abundances for the primary star. Spectral variations due to pulsational and rotational effects are analysed in detail in Sect. 5, while the identification of pulsational modes is discussed in Sects. 6 and 7. Finally, the main results are summarized in Sect. 8.

\section{Observations and data processing}

An overview of the spectroscopic material analysed in the present paper is given in Table 1. It includes (from large to small size of the data set):

- 646 spectra gathered with the spectrograph CORALIE (Queloz et al. 2000) mounted on the Swiss $1.2 \mathrm{~m}$ Leonhard Euler Telescope in La Silla (Chile).
- 12 observations taken with the High Accuracy Radial velocity Planet Searcher in spectropolametric mode (HARPS). This instrument is attached to the ESO $3.6 \mathrm{~m}$ telescope at the observatory in La Silla (Chile). Each observation consists of four independent subexposures that were treated as individual measurements, making 48 HARPS spectra in total. It should be noted that the covered wavelength range, as specified in Table 1, has a small gap of $10 \mathrm{~nm}$ at $\lambda 530 \mathrm{~nm}$.

- 27 spectra observed with the High Efficiency and Resolution Canterbury University Large Echelle Spectrograph (HERCULES). It is a fibre fed spectrograph connected to the $1 \mathrm{~m}$ McLellan telescope at the University of Canterbury Mt. John Observatory (New Zealand).

- 8 spectra taken with the Grating Instrument for Radiation Analysis with a Fibre Fed Échelle (GIRAFFE) in conjunction with the 1.9-m telescope of the South African Astronomical Observatory (SAAO) in Sutherland (South Africa).

- 2 spectra recorded with the Ultraviolet and Visual Echelle Spectrograph (UVES) mounted on UT2 of the Very Large Telescope (VLT) at the Paranal Observatory (Chile).

- 2 spectra observed with the Fiber-fed Extended Range Optical Spectrograph (FEROS) mounted on the MPG/ESO $2.2 \mathrm{~m}$ telescope at the La Silla observatory (Chile).

The raw spectra were subjected to the usual basic reduction process which consists of de-biasing, background subtraction, order extraction, flat-fielding from white lamp observations, and wavelength calibration by means of a thorium lamp. For the CORALIE spectra, the pipeline reduction software (Baranne et al. 1996) available at the telescope was used. The HARPS observations were reduced using the data reduction software available at the ESO headquarters in Germany and on La Silla. For the HERCULES spectra, it was done by using a MATLAB pipeline written by D.J. Wright. For the GIRAFFE spectra, these steps were performed within the ESO-MIDAS environment (version 12FEBpl1.1) with scripts compiled by P. De Cat. The UVES and FEROS spectra were reduced using standard IRAF tasks. All the spectra were shifted to the solar system barycentre rest frame and the Barycentric Julian Dates of midexposure were calculated.

Telluric lines were modelled and removed. Scaled telluric spectra were calculated using the TAPAS ${ }^{1}$ service (Bertaux et al. 2014). For the cosmic ray removal in large spectra sets, we proceed as follows. First we measured the preliminary radial velocities, shifted the spectra accordingly, and calculated an average spectrum. Then we subtracted the average spectrum and detected in the residual spectra the cosmic rays as pixels with count levels above five times the noise level. The flux value at those pixels were replaced in the original spectra by the corresponding value in the average spectrum. The CORALIE spectrograms showed a pseudo-periodic continuum modulation of about $0.5-1.0 \%$ of the continuum level. To improve the comparison with observations from other instruments, we modelled and fit this pattern. The data processing is illustrated in Fig. 1.

\section{A long-term binary}

From a visual inspection of radial velocities, a long-term variation is evident and we interpret it as being due to binary orbital motion. In addition, we confirm the dominant pulsation mode reported by Neiner et al. (2012a), we identify a second pulsation mode of $\beta$ Cep type, and we also derive a reliable rotation period for the primary. Here, we focus on our binarity analysis.

\footnotetext{
1 http://cds-espri.ipsl.fr/tapas/
} 
Table 1. Overview of the spectroscopic data of HD 96446 used in this study.

\begin{tabular}{lrrrcccccc}
\hline \hline Spectrograph & $\#$ & $\begin{array}{r}\text { Timespan } \\
\text { (days) }\end{array}$ & Begin date & End date & Resolution & $\begin{array}{c}\text { Begin } \lambda \\
(\mathrm{nm})\end{array}$ & $\begin{array}{c}\text { End } \lambda \\
(\mathrm{nm})\end{array}$ & $\begin{array}{c}\text { Exp. Time } \\
\mathrm{s}\end{array}$ & $S / N$ \\
\hline CORALIE & 646 & 116 & $11 / 02 / 2014$ & $07 / 06 / 2014$ & $50 \mathrm{k}$ & 387 & 690 & 600 & $60-110$ \\
HARPS & 48 & 1851 & $23 / 05 / 2011$ & $16 / 06 / 2016$ & $120 \mathrm{k}$ & 378 & 691 & $900-1800$ & $130-210$ \\
HERCULES & 27 & 3.08 & $08 / 04 / 2014$ & $11 / 04 / 2014$ & $45 \mathrm{k}$ & 403 & 1009 & 600 & $50-90$ \\
GIRAFFE & 8 & 0.06 & $10 / 04 / 2014$ & $10 / 04 / 2014$ & $35 \mathrm{k}$ & 422 & 622 & 600 & $30-50$ \\
UVES & 2 & 1.14 & $14 / 01 / 2006$ & $15 / 01 / 2006$ & $80 \mathrm{k}-110 \mathrm{k}$ & 303 & 854 & $50-100$ & $200-430$ \\
FEROS & 2 & 1.12 & $19 / 03 / 2009$ & $20 / 03 / 2009$ & $48 \mathrm{k}$ & 370 & 900 & 60 & $140-190$ \\
\hline
\end{tabular}

Notes. It includes the name of the spectrograph, the number of spectra (\#), the total time span (in days), the begin date and end date of the observations, the begin and end wavelength $\lambda$ of the observed range (in $\mathrm{nm}$ ), and the typical S/N.

In Sect. 4 we present a sophisticated spectral analysis with a model atmosphere. In Sect. 5 we describe our analysis of the other physical phenomena involved, namely rotational modulation and stellar pulsation after a removal of the orbital motion in the spectra.

\subsection{Radial velocity measurements}

Our binary model is based on radial velocity data. They were measured for all available spectra by means of cross-correlations using the average of all HARPS spectra of our object as a template. The template velocity was determined by cross-correlation against a synthetic spectrum. The spectral regions used in the cross-correlations excluded $\mathrm{H}_{\mathrm{I}}$ lines and strong $\mathrm{He}_{\mathrm{I}}$ lines.

All instruments employed in our study are high-resolution spectrographs with a radial velocity precision of the order of $100 \mathrm{~m} \mathrm{~s}^{-1}$ or better, except GIRAFFE for which radial velocities have uncertainties of about half a $\mathrm{km} \mathrm{s}^{-1}$. Since the radial velocity variations to be measured are very small $\left(\sim 10^{2}-10^{3} \mathrm{~m} \mathrm{~s}^{-1}\right)$, all the radial velocity measurements have to be determined in the same way and to be accurately referenced to the same system. Most of the spectrographs used here are very stable. However, there are small systematic differences between the different instruments. For instance, by measuring the interstellar Na I doublet at $25889-94 \AA$, we found a mean difference of $0.86 \pm 0.01 \mathrm{~km} \mathrm{~s}^{-1}$ between the HARPS and CORALIE observations $\left(\sigma_{\text {HARPS }}=0.013 \mathrm{~km} \mathrm{~s}^{-1}, \sigma_{\text {CORALIE }}=0.19 \mathrm{~km} \mathrm{~s}^{-1}\right)$. The measurement of the same lines for FEROS and HERCULES agrees with CORALIE within $\sim 0.1 \mathrm{~km} \mathrm{~s}^{-1}$.

For our variability analysis of the radial velocities, we used only the observations for which the zero point can reliably be established as an additional free parameter during the velocitycurve fitting. Interstellar lines cannot be used to correct individual spectra since in most cases the signal-to-noise ratio $(\mathrm{S} / \mathrm{N})$ is not high enough to measure radial velocities from one or a few spectral lines. For this reason we decided to analyse the velocity variations using only the CORALIE, HARPS, and HERCULES data sets, which have high internal precision and a number of observations large enough to determine the instrumental zero points. The remaining spectra (FEROS, UVES, and GIRAFFE) were corrected measuring $\mathrm{Na}$ I and/or Ca II interstellar lines in the average spectrum of each data set, but were used only to check the long-term consistency of the periodical variations.

The obtained radial velocities are available at the CDS (see Sect. 3.2). The consigned errors are formal uncertainties provided by the IRAF task fxcor used to perform the crosscorrelations. This error corresponds mainly to the spectrum noise and does not include other uncertainties like systematic errors

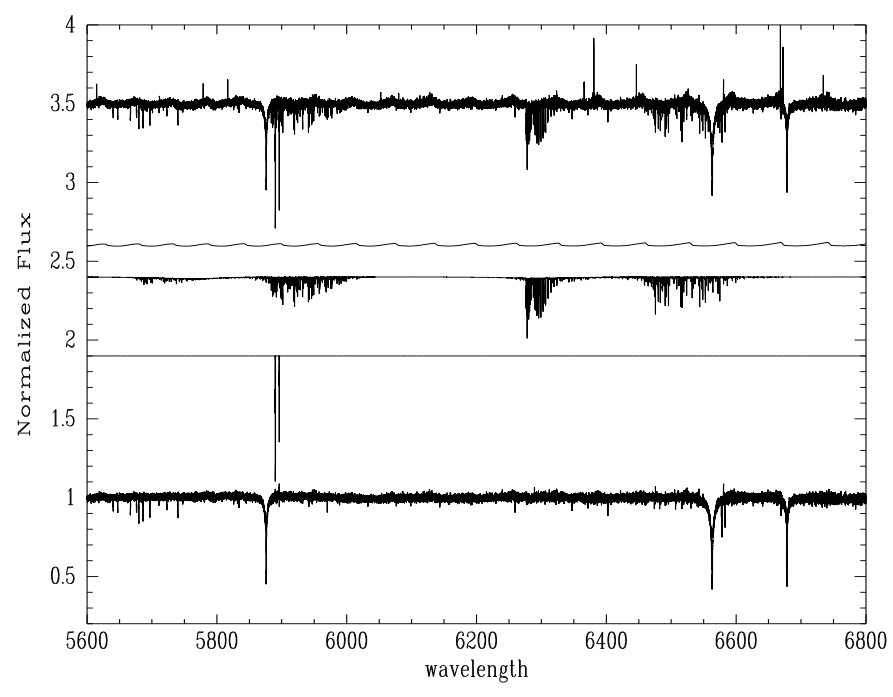

Fig. 1. Processing of CORALIE data. From top to bottom: raw spectrum, modelled fringing pattern, telluric spectrum, interstellar spectrum, corrected spectrum.

related to the instrumental zero point or to the use of different spectral regions for the cross-correlations.

\subsection{Radial velocity curve fitting}

We modelled the measured radial velocities using a Keplerian orbit plus three other periodic functions representing two pulsational modes and the rotational modulation

$\mathrm{RV}=f_{1}+f_{2}+f_{\mathrm{o}}+f_{\mathrm{r}}$,

where $f_{\mathrm{o}}$ is the binary orbital solution,

$f_{1}=\alpha_{1} \sin 2 \pi \phi_{1}+\beta_{1} \sin 4 \pi \phi_{1}$,

$f_{2}=\alpha_{2} \sin 2 \pi \phi_{2}$, and

$f_{\mathrm{r}}=\alpha_{\mathrm{rot}} \sin 2 \pi \phi_{\mathrm{rot}}$.

The phases $\phi_{1}, \phi_{2}$, and $\phi_{\text {rot }}$ are calculated with the pulsational periods $P_{1}$ and $P_{2}$, and the rotation period $P_{\text {rot }}$, respectively (Sect. 5). The second term in $f_{1}$ was included in order to describe appropriately the small assymetry of the radial velocity curve for this frequency.

Since the radial velocity variations related to the rotational velocity are very small, the rotational period was kept fixed at the value found by means of our spectrosocopy in combination with the spectropolarimetry of Järvinen et al. (2017) (see Sect. 5.1). 


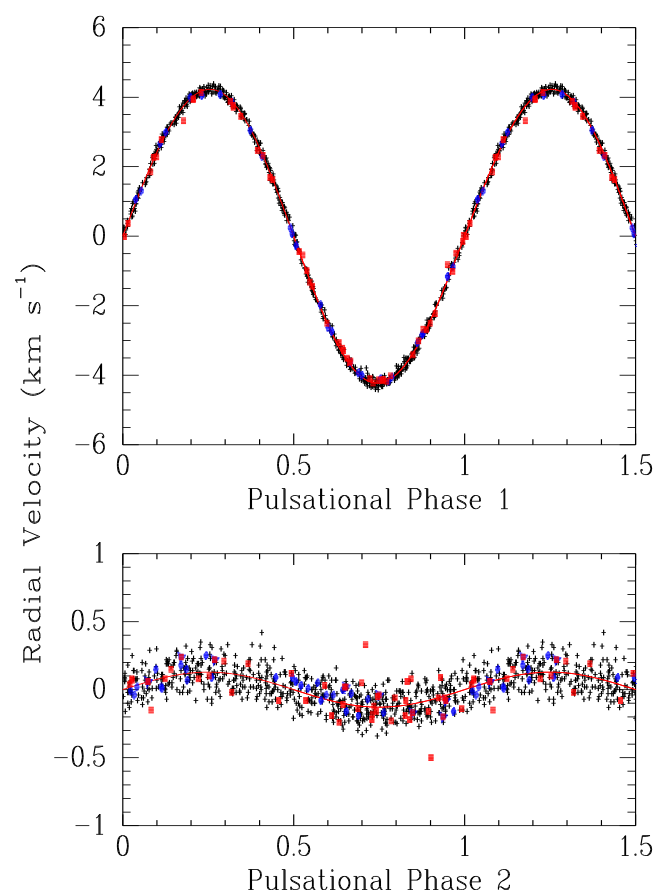

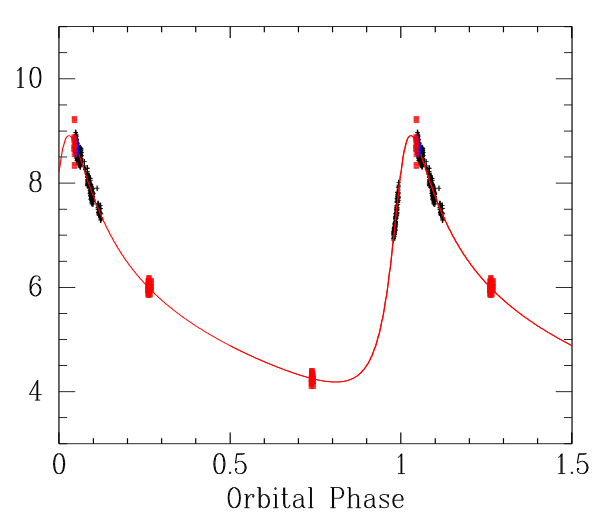

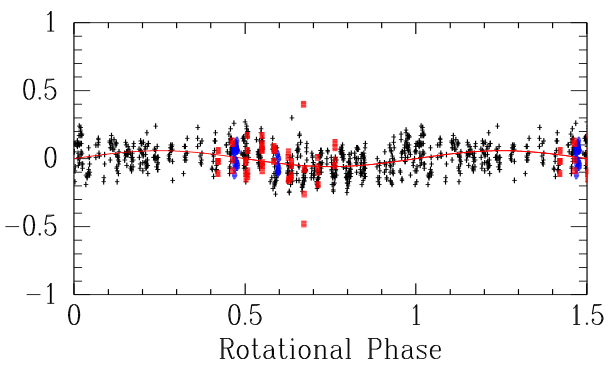

Fig. 2. Radial velocity curves. In each panel we show the variations corresponding to a specific effect. Left panels: pulsational variations. Right panels: orbital motion (top), and rotational modulation (bottom). Black: CORALIE, red: HARPS, blue: HERCULES.
In the calculation of the phases, the barycentric Julian dates of the observations were corrected for the light-time effect to take the time shifts related with the variations of the distance to the observer during the orbital cycle into account.

The obtained radial velocity curves are shown in Fig. 2. Each panel shows one of the four periodic variations present in this system. In a given panel the points represent the observed velocity minus the calculated curves shown in the remaining three panels. The rms of the residuals in this final fit is $0.10 \mathrm{~km} \mathrm{~s}^{-1}$. Table 2 lists the parameters of the radial velocity curves. The velocity amplitudes $\alpha_{1}, \beta_{1}, \alpha_{2}$, and $\alpha_{\text {rot }}$ are defined by Eqs. (2)-(4). The orbital parameters are listed using usual symbols $\left(V_{\gamma}, P_{\text {orb }}, a_{1} \sin i, e, \omega\right)$. The origin of orbital phases $T_{\text {orb }}$ is the periastron passage. Stellar rotation has little impact on the radial velocity variations, so the rotational ephemeris will be determined in Sect. 5 on the basis of the variation in spectral line intensities and magnetic fields. The radial velocity data are in Table 3, available at the CDS. The columns in this table include the pulsational, orbital, and rotational phases as well as the calculated velocity for each effect contributing to the observed RV variation.

\subsection{Secondary component}

Despite the large number of spectra available, the small amplitude of the radial velocity variations makes it impossible to detect the secondary component by disentangling techniques based on Doppler shifts. However, we were able to discover the secondary features by averaging all CORALIE observations corrected by the radial velocity of the primary star. In this master spectrum, the lines of the primary remained sharp, and were easily distinguishable from the secondary line set, which has a different morphology (later spectral type) and a larger rotational broadening. The extra broadening added to the secondary lines as a consequence of the different radial velocity values of this component in the spectra that were combined, had little impact. The estimated distribution of the radial velocity of the secondary in these spectra has an rms of $\sim 2.5 \mathrm{~km} \mathrm{~s}^{-1}$, while its projected rotational velocity is $v \sin i \approx 55 \mathrm{~km} \mathrm{~s}^{-1}$. Figure 3 shows small
Table 2. Parameters of the radial velocity curves.

\begin{tabular}{lcc}
\hline \hline$T_{1}$ & & $2456760.02435 \pm 0.00002$ \\
$V_{\gamma}$ & $\mathrm{km} \mathrm{s}^{-1}$ & $5.57 \pm 0.02$ \\
$\alpha_{1}$ & $\mathrm{~km} \mathrm{~s}^{-1}$ & $4.223 \pm 0.005$ \\
$\beta_{1}$ & $\mathrm{~km} \mathrm{~s}^{-1}$ & $-0.066 \pm 0.005$ \\
$T_{2}$ & & $2456760.0786 \pm 0.0008$ \\
$\alpha_{2}$ & $\mathrm{~km} \mathrm{~s}^{-1}$ & $0.13 \pm 0.01$ \\
$P_{1}$ & $\mathrm{~d}$ & $0.092919828 \pm 0.000000009$ \\
$P_{2}$ & $\mathrm{~d}$ & $0.1108037 \pm 0.0000004$ \\
$T_{\text {orb }}$ & & $2456717.3 \pm 0.7$ \\
$a_{1} \sin i$ & $\mathrm{AU}$ & $0.1410 \pm 0.0010$ \\
$\omega$ & $\mathrm{rad}$ & $5.495 \pm 0.017$ \\
$e$ & & $0.586 \pm 0.006$ \\
$P_{\text {orb }}$ & $\mathrm{d}$ & $799.3 \pm 1.7$ \\
$T_{\text {rot }}$ & & $2456604.6 \pm 0.5$ \\
$\alpha_{\text {rot }}$ & $\mathrm{km} \mathrm{s}{ }^{-1}$ & $0.06 \pm 0.01$ \\
$P_{\text {rot }}$ & $\mathrm{d}$ & 23.38 (fixed) \\
\hline
\end{tabular}

spectral windows where secondary lines are clearly visible. For comparison, synthetic spectra of $T_{\text {eff }}=9500$ and $10000 \mathrm{~K}$, scaled by a factor 0.05 and 0.06 , respectively, are overplotted. The pixel-to-pixel $\mathrm{S} / \mathrm{N}$ achieved in the master spectrum is about 2000 , which allowed us to detect secondary lines with core intensity between 0.002 and 0.006 of the binary continuum level. The intensity of $\mathrm{Ti}$ II and $\mathrm{Mg}$ I lines with respect to Fe II suggests a spectral type A0.

To estimate the mass ratio we used the fact that all CORALIE observations are taken in a small range of orbital phases. The measured velocity of the secondary in the master spectrum is $-3.1 \pm 1.2 \mathrm{~km} \mathrm{~s}^{-1}$, while the mean velocity of the primary in the 646 spectra is $8.08 \mathrm{~km} \mathrm{~s}^{-1}$ and the centre-of-mass velocity is $5.57 \mathrm{~km} \mathrm{~s}^{-1}$. From these values we estimated the mass-ratio to be $q=0.29 \pm 0.04$. This value is consistent with the fluxratio according to stellar models. For an isochrone of $10 \mathrm{Myr}$ in the Geneva stellar models for example, the mass ratio of two 

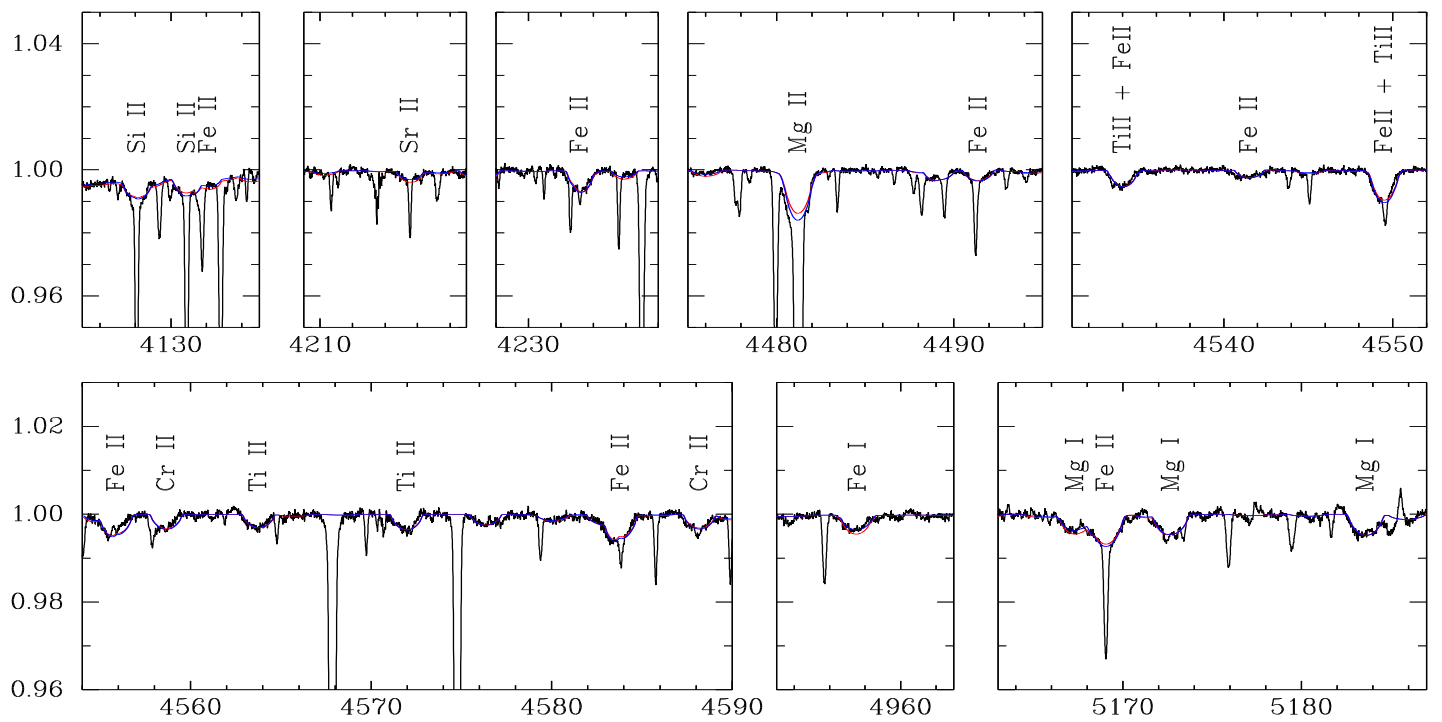

Fig. 3. Line spectrum of the faint binary companion. In selected spectral windows, the average of all 646 CORALIE spectra (corrected by radial velocity) is plotted along with two synthetic spectra of $T_{\text {eff }}=9500 \mathrm{~K}$ (red) and $T_{\text {eff }}=10000 \mathrm{~K}$ (blue) appropriately scaled.

stars of $T_{\text {eff }}=22000 \mathrm{~K}$ and $10000 \mathrm{~K}$ is 0.275 . A rough estimate of the stellar masses $\left(M_{1} \approx 8.7-9.3 M_{\odot}, M_{2} \approx 2.1-2.8 M_{\odot}\right.$, $q=0.29 \pm 0.04)$ is enough to constrain the orbital inclination of the system. Using these values and the spectroscopic value of $a_{1} \sin ^{3} i$, we obtain the orbital inclination to be $i_{\text {orb }}=33.4 \pm 1.4^{\circ}$.

\section{Model atmosphere and spectral analysis}

\subsection{Codes and computations}

We have determined atmospheric and fundamental parameters and chemical abundances of HD 96446 following the methodology proposed by Przybilla et al. (2016), which was also applied to other He-rich stars with magnetic fields by González et al. (2017) and Castro et al. (2017). In brief, the spectral analysis was based on a hybrid non-LTE approach (Nieva \& Przybilla 2007, 2012; hereafter NP12). Model atmospheres were computed with ATLAS9 (Kurucz 1993), which assumes planeparallel geometry, homogeneity, and hydrostatic, radiative, and local thermodynamic equilibrium (LTE). Non-LTE level populations and model spectra were calculated on the pre-computed atmospheric structures with recent versions of DETAIL and SURFACE (Giddings 1981; Butler \& Giddings 1985). The coupled radiative transfer and statistical equilibrium equations were solved with DETAIL, employing the accelerated lambda iteration scheme of Rybicki \& Hummer (1991), which allows complex ions to be treated in a realistic way. Synthetic spectra were calculated with SURFACE, using refined line-broadening theories. Continuous opacities due to $\mathrm{H}$ and $\mathrm{He}$ were considered in non-LTE, and line blocking was accounted for via LTE opacity sampling, employing the method of Kurucz (1996).

Dedicated computations were required to constrain the atmospheric parameters and elemental abundances of HD 96446 because its high He content is not considered in pre-existing spectral model grids. Non-LTE level populations and synthetic spectra for a dozen chemical elements were calculated using model atoms listed in Table 4. The table summarizes the number of explicit non-LTE levels in the different ionization stages, the number of radiative bound-bound transitions and the reference to the model atom. Typically, the levels are terms, and the transitions are multiplet transitions. In some cases additional
Table 4. Model atoms for non-LTE calculations.

\begin{tabular}{llll}
\hline \hline Ion & Levels & Transitions & Reference \\
\hline $\mathrm{H}$ & 20 & 190 & {$[1]$} \\
$\mathrm{He}_{\text {I/II }}$ & $29+6 / 20$ & $162 / 190$ & {$[2]$} \\
$\mathrm{C}_{\text {II } / \text { III }}$ & $68 / 70$ & $425 / 373$ & {$[3]$} \\
$\mathrm{N}_{\text {II }}$ & 77 & 462 & {$[4]$} \\
$\mathrm{O}_{\text {I/II }}$ & $51 / 52$ & $243 / 134$ & {$[5]$} \\
$\mathrm{Ne}_{\text {I }}$ & 153 & 952 & {$[6]$} \\
$\mathrm{Mg}_{\text {II }}$ & 37 & 236 & {$[7]$} \\
$\mathrm{Al}_{\text {III }}$ & $46+1$ & 272 & {$[8]$} \\
$\mathrm{Si}_{\text {II } / \text { III/IV }}$ & $52+3 / 68+4 / 33+2$ & $357 / 572 / 242$ & {$[9]$} \\
$\mathrm{S}_{\text {II/III }}$ & $78 / 21$ & $302 / 34$ & {$[10]$} \\
$\mathrm{Ar}_{\text {II }}$ & 56 & 596 & {$[11]$} \\
$\mathrm{Fe}_{\text {II } / \text { III }}$ & $265 / 60+46$ & $2887 / 2446$ & {$[12]$}
\end{tabular}

References. [1] Przybilla \& Butler (2004); [2] Przybilla (2005); [3] Nieva \& Przybilla (2006, 2008); [4] Przybilla \& Butler (2001); [5] Przybilla et al. (2000), Becker \& Butler (1988); [6] Morel \& Butler (2008); [7] Przybilla et al. (2001); [8] Przybilla (in prep.); [9] Przybilla \& Butler (in prep.); [10] Vrancken et al. (1996); [11] Butler (in prep.); [12] Becker (1998), Morel et al. (2006), see text.

"superlevels" were considered (indicated by the plus sign), which were packed over many levels. Additional ionization stages were also considered, typically consisting either of the ground state of the next higher ionization stage only, or of models of low complexity. In the case of $\mathrm{Ne}$, the levels are finestructure components. Several of the model atoms have recently been updated, mostly by consideration of improved oscillator strengths and ab initio collisional data. We note that the original input data for line-formation computations of Fe III by Morel et al. (2006) were corrected as described by NP12.

Fits of model spectra to the observations were performed with the Spectrum Plotting and Analysing Suite (SPAS, Hirsch 2009), which uses the downhill simplex algorithm of Nelder \& Mead (1965) to minimize $\chi^{2}$. The code allows interpolation between model grid points for up to three parameters simultaneously. Instrumental, rotational, and (radial-tangential) macrobroadening functions were applied to the theoretical profiles. 


\subsection{Spectral line analysis and results}

Balmer lines, $\mathrm{He} \mathrm{I} / \mathrm{II}$ lines ${ }^{2}$, and ionization equilibria ${ }^{3}$ of $\mathrm{C}$ II/III,

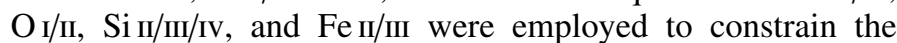
atmospheric parameters - effective temperature $T_{\text {eff }}$, (logarithmic) surface gravity $\log g$, microturbulence $\xi$, helium abundance $y$ (by number), projected rotational velocity $v \sin i$, and macroturbulent velocity $\zeta$ - using an approach similar to that of NP12. We analysed the averaged CORALIE spectrum for this, plus the co-added FEROS spectra in case of the O I $\lambda \lambda 7771-5 \AA$ triplet. Once the atmospheric parameters were fixed, elemental abundances for the additional chemical species were determined.

The atmospheric parameters and chemical abundances derived here are listed in Table 5. Furthermore, the table summarizes general information on the star, such as the spectral type, the systemic radial velocity $v_{\text {rad,sys }}$, the spectroscopic distance $d_{\text {spec }}$ (see NP12), and the magnetic dipole field strength $B_{\mathrm{d}}$ (Järvinen et al. 2017). Figure 4 shows a comparison of our bestfit global spectrum synthesis to parts of the averaged CORALIE observations. Overall, a good to excellent fit is achieved, resulting in a simultaneous match of the hydrogen and helium lines, and of the ionization equilibria.

A very high helium abundance is already qualitatively supported by the presence of much broader $\mathrm{He}_{\mathrm{I}}$ lines than in the other He-strong stars investigated by us previously, CPD $-57^{\circ} 3509$ (Przybilla et al. 2016), CPD -62 2124 (Castro et al. 2017), and HD 164492Cb (González et al. 2017), which have $y$ in the range of $0.28-0.35$. Also, the forbidden He I components ${ }^{4}$ (e.g. at $\lambda \lambda 4045$ and $4911 \AA$ ), unobserved in He-normal B-stars and inconspicuous in typical He-strong stars, are very pronounced (see Fig. 4), similar to the case of extreme He-stars (e.g. Przybilla et al. 2005; Kupfer et al. 2017). The quantitative analysis yields $y=0.60 \pm 0.03$ (or 0.86 by mass), more than twice the value proposed by Neiner et al. (2012a), and to our knowledge the highest found among the He-strong stars so far. The particular geometry with a large He-rich spot always present in the visible hemisphere (see below) may be important in this context.

Metal abundances are consistent to typically better than a factor of 2 with the cosmic abundance standard (CAS) derived from early B-type stars in the solar neighbourhood, as proposed by NP12 and extended by Przybilla et al. (2013), if measured relative to hydrogen (which is significantly depleted). This supports the view that the chemical peculiarity of the He-strong stars results from a weak fractionated stellar wind, where metal ions drive the wind and drag along the ionized hydrogen because of Coulomb coupling, while the neutral helium falls back and accumulates in the stellar photosphere (Hunger \& Groote 1999; Krtička \& Kubát 2001). Notable is the N/C abundance ratio, which is higher than that of CAS. This may be an indicator of mixing of the surface layers with $\mathrm{CN}$-processed material from the core, as found in some magnetic stars (see e.g. Nieva \& Przybilla 2014). However, a decisive statement cannot be made on this because of the overall abundance peculiarities.

The respective LTE model is also plotted in Fig. 4 in order to show the extent of non-LTE effects on the line-formation computations. While non-LTE effects are of minor importance to the hydrogen lines (with the exception of $\mathrm{H} \alpha$ ) and many blue helium

\footnotetext{
2 Despite the relatively low surface temperature of the star, the He II $4686 \AA$ line is present because of the high He content.

3 Abundances of different ionization stages of an element agree within the uncertainties for a given set of atmospheric parameters.

4 They are not modelled, as these components are not implemented in the available broadening tables (also in the case of the $\lambda 4144 \AA$ line).
}

Table 5. Parameters and elemental abundances of the primary star of the system HD 96446.

\begin{tabular}{|c|c|c|}
\hline Sp. Type & B2 IV He-strong & \\
\hline$v_{\text {rad,sys }}$ & $5.57 \pm 0.02 \mathrm{~km} \mathrm{~s}^{-1}$ & \\
\hline$d_{\text {spec }}$ & $741 \pm 97 \mathrm{pc}$ & \\
\hline$B_{\mathrm{d}}$ & $4.64 \pm 0.88 \mathrm{kG}$ & \\
\hline \multicolumn{3}{|c|}{ Atmospheric parameters: } \\
\hline$T_{\text {eff }}$ & $21800 \pm 300 \mathrm{~K}$ & \\
\hline $\log g(\mathrm{cgs})$ & $3.85 \pm 0.10$ & \\
\hline$y$ (number fraction) & $0.60 \pm 0.03$ & \\
\hline Microturb. vel. $\xi$ & $6 \pm 1 \mathrm{~km} \mathrm{~s}^{-1}$ & \\
\hline$v \sin i$ & $2 \pm 1 \mathrm{~km} \mathrm{~s}^{-1}$ & \\
\hline Macroturb. vel. $\zeta$ & $2 \pm 1 \mathrm{~km} \mathrm{~s}^{-1}$ & \\
\hline \multicolumn{3}{|c|}{ Non-LTE metal abundances: } \\
\hline & HD 96446 & CAS \\
\hline $\log (\mathrm{C} / \mathrm{H})+12$ & $8.24 \pm 0.06(13)$ & $8.33 \pm 0.04$ \\
\hline $\log (\mathrm{N} / \mathrm{H})+12$ & $7.97 \pm 0.09(57)$ & $7.79 \pm 0.04$ \\
\hline $\log (\mathrm{O} / \mathrm{H})+12$ & $8.56 \pm 0.05(53)$ & $8.76 \pm 0.05$ \\
\hline $\log (\mathrm{Ne} / \mathrm{H})+12$ & $8.14 \pm 0.07(13)$ & $8.09 \pm 0.05$ \\
\hline $\log (\mathrm{Mg} / \mathrm{H})+12$ & $7.31 \pm 0.03(2)$ & $7.56 \pm 0.05$ \\
\hline $\log (\mathrm{Al} / \mathrm{H})+12$ & $6.23 \pm 0.08(4)$ & $6.30 \pm 0.07$ \\
\hline $\log (\mathrm{Si} / \mathrm{H})+12$ & $7.19 \pm 0.08(13)$ & $7.50 \pm 0.05$ \\
\hline $\log (\mathrm{S} / \mathrm{H})+12$ & $7.24 \pm 0.04(23)$ & $7.14 \pm 0.06$ \\
\hline $\log (\operatorname{Ar} / \mathrm{H})+12$ & $6.50 \pm 0.02(6)$ & $6.50 \pm 0.08$ \\
\hline $\log (\mathrm{Fe} / \mathrm{H})+12$ & $7.35 \pm 0.09(27)$ & $7.52 \pm 0.03$ \\
\hline \multicolumn{3}{|l|}{ Photometric data: } \\
\hline$V$ & $6.686 \pm 0.005$ & \\
\hline$B-V$ & $-0.138 \pm 0.020$ & \\
\hline$E(B-V)$ & $0.060 \pm 0.003$ & \\
\hline$M_{V}$ & $-2.85 \pm 0.29$ & \\
\hline$M_{\mathrm{bol}}$ & $-4.94 \pm 0.29$ & \\
\hline \multicolumn{3}{|c|}{ Fundamental parameters: } \\
\hline$M / M_{\odot}$ & $9.1 \pm 0.3$ & \\
\hline$R / R_{\odot}$ & $6.1 \pm 0.9$ & \\
\hline $\log L / L_{\odot}$ & $3.87 \pm 0.11$ & \\
\hline$\tau$ & $23.4_{-1.0}^{+1.1} \mathrm{Myr}$ & \\
\hline$\tau / \tau_{\mathrm{MS}}$ & $0.77_{-0.04}^{+0.03}$ & \\
\hline
\end{tabular}

Notes. $1 \sigma$ uncertainties are given. For abundances they are from the line-to-line scatter; systematic errors amount to an additional $\sim 0.15$ dex. The number of lines considered for the abundance determination is given in brackets. The cosmic abundance standard (CAS, NP12) in the solar neighbourhood is given for reference, along with data for $\mathrm{Al}, \mathrm{S}$, and Ar from Przybilla et al. (2013).

lines, the red helium lines are considerably affected, and nonLTE effects are crucial for establishing ionization equilibria. For example, the weak Si II lines (equivalent widths of $\sim 5-20 \mathrm{~m} \AA$ ) become significantly weaker in non-LTE, while the strong Si III and the weak Si Iv lines become even stronger. Many metal lines are affected and it is not possible to easily transfer experiences made with He-normal stars to the He-strong case. The reason is that pronounced hydrogen continuous opacities are replaced by much smaller helium continuum opacities, leading to a boosting of the relative line-to-continuum opacities and to an overall hardening of the radiation field in the He-rich case (Kupfer et al. 2017). The effects on collision rates would require detailed investigations, as on the one hand the higher mean molecular weight of the He-rich composition leads to increased densities, while on the other hand the higher ionization potential of helium results in a reduction of free electrons. In consequence, non-LTE effects tend to strengthen in He-rich environments. 


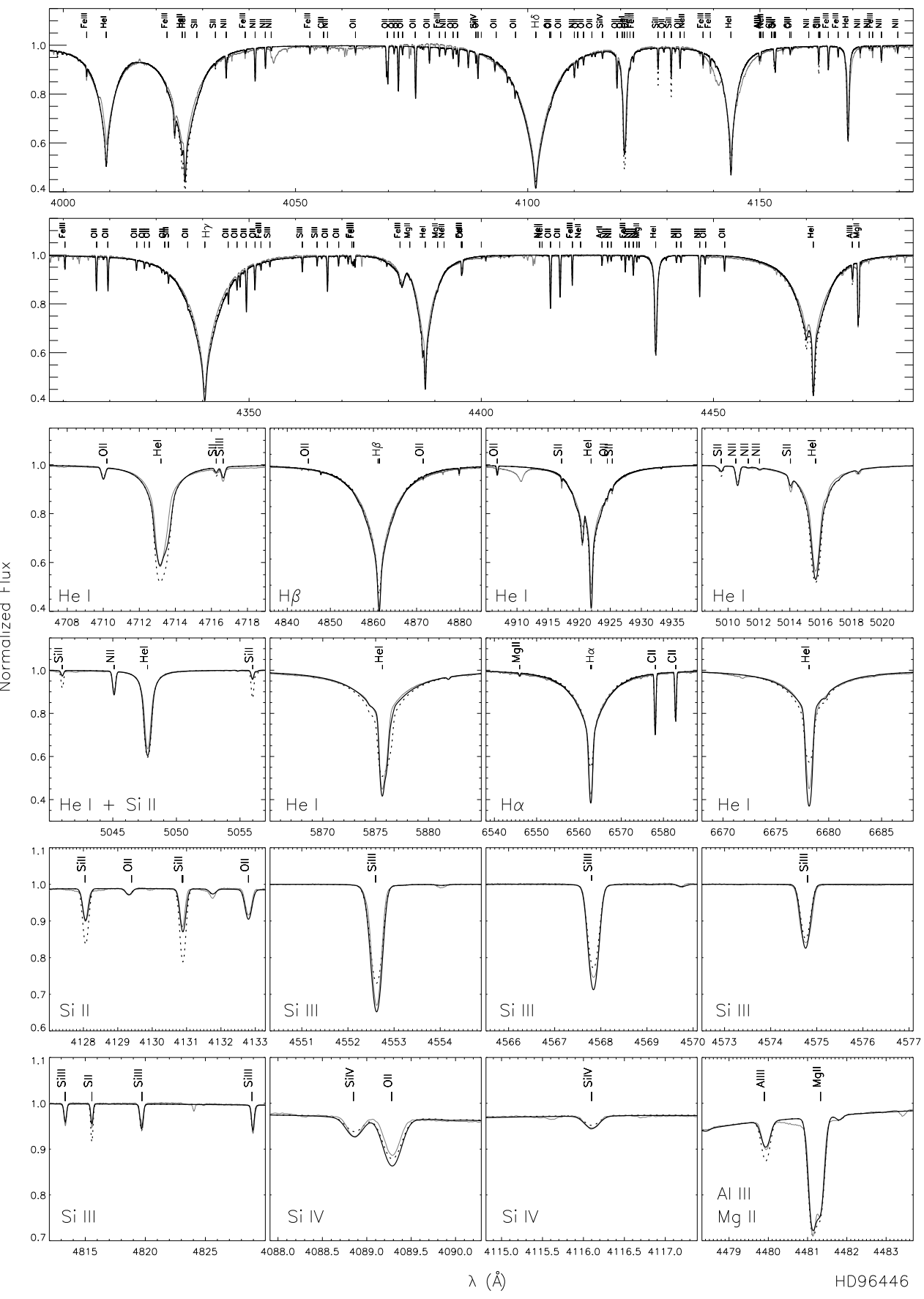

Fig. 4. Comparison between global synthetic non-LTE spectrum (black line) and observed spectrum (grey line) for HD 96446 for a range of diagnostic features. The two top panels concentrate on two extended regions in the blue part of the spectrum, the two middle panels on other important hydrogen and helium lines, and the two bottom panels on an example for a ionization equilibrium, $\mathrm{Si}$ II-IV. The corresponding LTE spectrum synthesis is indicated by a dotted line.
The $T_{\text {eff }}$ determination can be verified by a comparison of the observed and computed spectral energy distribution (SED; see Fig. 5). Spectrophotometry as obtained with the International Ultraviolet Explorer (IUE; exposures SWP05764 and LWR05000) were extracted from the MAST archive. Wide-band photometry in the Johnson $U B V$ passbands was adopted from (Mermilliod 1997, see Table 5) and in the 2MASS JHK passbands from Cutri et al. (2003). The magnitudes were converted to fluxes using the zero points described by Heber et al. (2002). This facilitated the reddening $E(B-V)$ to be determined, and consequently the absolute visual and bolometric magnitudes, $M_{\mathrm{V}}$ and $M_{\mathrm{bol}}$, respectively, see also Table 5.

Finally, the fundamental stellar parameters of mass $M$, radius $R$, luminosity $L$, evolutionary age $\tau$, and fractional main-sequence lifetime $\tau / \tau_{\text {MS }}$ were derived via comparison with Geneva stellar evolution models (Ekström et al. 2012), as summarized in the last block of Table 5. The location of the star in the $\log T_{\text {eff }}-$ $\log g$ plane with respect to the evolutionary tracks and isochrones (see Fig. 6) provided $M, \tau$, and $\tau / \tau_{\mathrm{MS}}$, respectively. Luminosity and radius followed, once the spectroscopic distance was determined. We want to mention that the spectroscopic distance is greater and therefore the luminosity are higher than those indicated by conversion of the Hipparcos or Gaia parallaxes into a distance, as usually done in the literature. Because the relative error of the HIPPARcos parallax $\Delta \pi / \pi$ for HD 96446 is larger than $10 \%$, second-order effects become important and the interpretation of the derived quantities more complicated, and as a general rule the derived parameters should not be used (van Leeuwen 2007, p. 86). The parallax for HD 96446 in the Gaia Data Release 2 (DR2, Gaia Collaboration 2018) should also be viewed with 


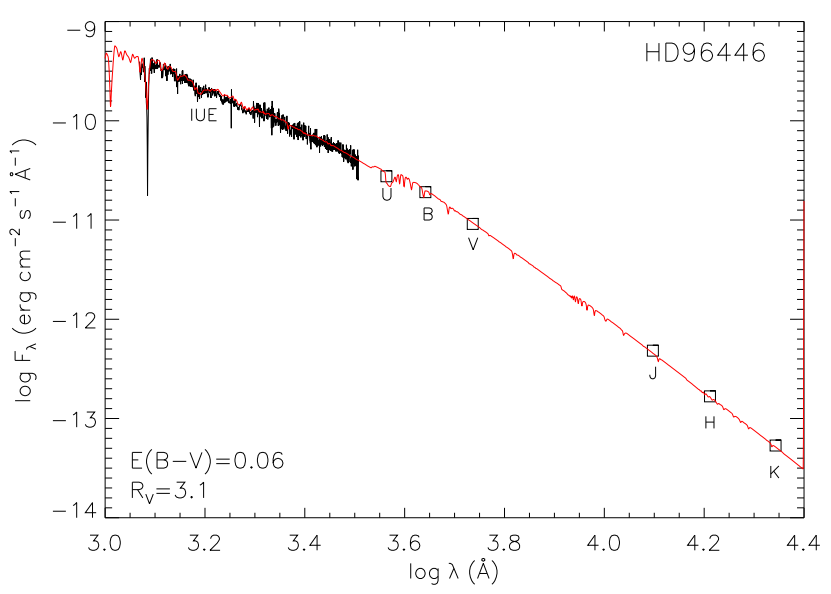

Fig. 5. Comparison of the observed spectral energy distribution of HD 96446 (black line, squares) with that of the ATLAS9 model (red).

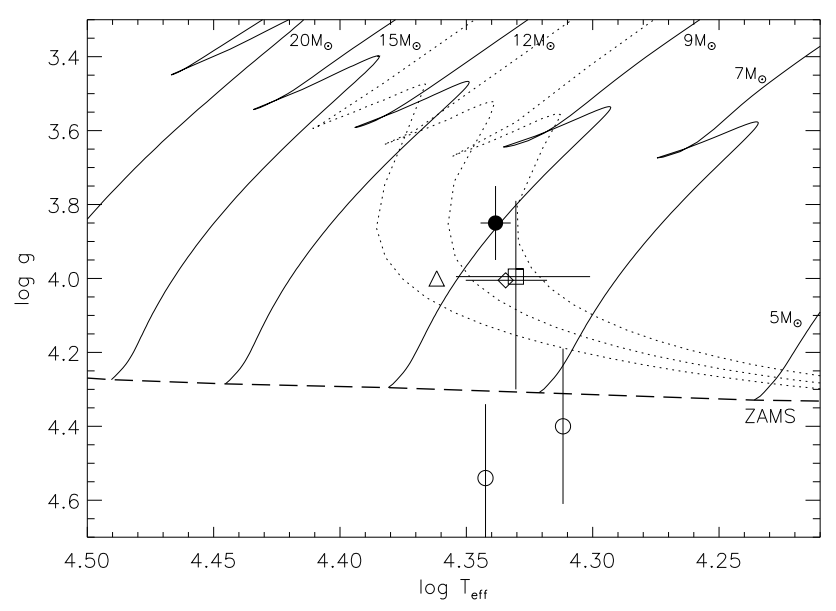

Fig. 6. HD 96446 in the $\log T_{\text {eff }}-\log g$ plane. Our solution (black dot) is compared to previous atmospheric parameter determinations reported in the literature: Hunger \& Groote (1999, box), Zboril et al. (1997, circles), Zboril \& North (2000, triangle), and Neiner et al. (2012a, diamond). Where available, $1 \sigma$ error bars are shown. Overlayed are Geneva evolution tracks for rotating stars, computed for metallicity $Z=0.014$ and an initial value of $40 \%$ of the critical rotation rate (Ekström et al. 2012, full lines). Corresponding isochrones for $\log \tau(\mathrm{Myr})=7.2,7.3$, and 7.4 are also shown (dotted lines, from left to right), and the position of the zero age main sequence (long dashed line).

caution because of the binary nature of this star and the short time coverage of the Gaia DR2. The high value of "astrometric excess noise" for this star, 0.29 mas with a high significance, indicates that there is a highly significant problem with the astrometric solution. We therefore disregard inferences made from the HiPPARcos and Gaia parallaxes in the present work. These inferences will have to wait a future Gaia data release.

Limitations. Our analysis is based on the mean CORALIE spectrum, which assures that the results are representative of the mean properties of HD 96446. However, several factors are not considered in our modelling that are of importance for the real star. There are small spectral variations along the rotational cycle (chemical spots) and through the pulsational cycle (temperature variations), implying deviations from homogeneity. We also do not consider the effects of the magnetic field on the radiative transfer, all of which limit our ability to fit individual spectral lines in very detail. They comprise, in particular, signatures of Zeeman splitting and strengthening of some lines with unresolved Zeeman signatures, as reported for HD 96446 and investigated in LTE by Neiner et al. (2012a). This additional broadening was qualitatively compensated by the choice of a rather high value for the microturbulent velocity for this kind of star. Improved calculation will need to account for polarized non-LTE radiative transfer calculations, which are far beyond the scope of the present paper. Overall, we estimate that the systematic uncertainties in mean metal abundances caused by this broadening and the typical error bars for atmospheric parameters and uncertainties in the atomic data are somewhat higher than in normal early B-type stars, to a conservative $\sim 0.15$ dex.

\subsection{Comparison to previous work}

HD 96446 was subject to several investigations in the past. Atmospheric parameters were determined by Zboril et al. (1997), Hunger \& Groote (1999), Zboril \& North (2000), and Neiner et al. (2012a). The last two studies also provided metal abundances. The distribution in the $\log T_{\text {eff }}-\log g$ plane is visualized in Fig. 6. A wide scatter is found as for other He-strong stars (see Przybilla et al. 2016 for a discussion). Our solution implies that HD 96446 is more evolved than thought previously.

Concerning elemental abundances, Zboril \& North (2000) derive $y=0.34 \pm 0.005$, a $1 / 15$ solar carbon abundance, and $\mathrm{N}$ and $\mathrm{O}$ underabundant by factors of $\sim 2$ and 3 relative to solar. Neiner et al. (2012a) find $y=0.29_{-0.12}^{+0.09}$ and metal abundances (elemental coverage as in Table 5, except for neon) systematically depleted by a factor of $\sim 2.5-5$ below solar (which are similar to CAS values). Both studies therefore imply much lower helium abundances and an overall metal-poor composition when compared to the present work. Three main factors likely contribute to the differences: (i) the atmospheric parameters, in particular the large differences in helium abundance; (ii) the neglect of non-LTE effects in the previous studies; and (iii) the differences in oscillator strengths.

A comparison of the quality of line fits as displayed in Fig. 10 of Neiner et al. (2012a) and in our Fig. 4 is instructive in this context. The combination of the assumption of LTE and the lower helium abundance lead Neiner et al. (2012a) to propose a vertical stratification of the helium abundance to explain the mismatch of the fits for wings and cores of the observed helium lines. Our model matches wings and cores without the need for this ${ }^{5}$. In terms of neglected non-LTE effects we can compare the Neiner et al. profile fits for Si II $\lambda 5041 \AA$ and Si III $\lambda 4716 \AA$ with ours for an example. Silicon turns out to be one of the species affected most by non-LTE effects.

\section{Rotational modulation and stellar pulsation}

Several factors can cause line profile variations in HD 96446. First, the line spectrum of the moving binary companion appears as morphological distortions since its lines have not been removed. The expected variations within the CORALIE data set are, however, very small ( $\sim 0.001$ of the continuum level). In any case, the great difference in the timescales of the various phenomena together with the large number of spectra, give

5 There are small discrepancies between observations and our model in the cores of the strongest He I lines. Instead of vertical stratification, which is probably difficult to sustain in the face of mass-loss rates of the order of several $10^{-9}-10^{-8} M_{\odot} \mathrm{yr}^{-1}$, we suggest that unaccounted for non-LTE effects on the outer atmospheric structure are responsible. 

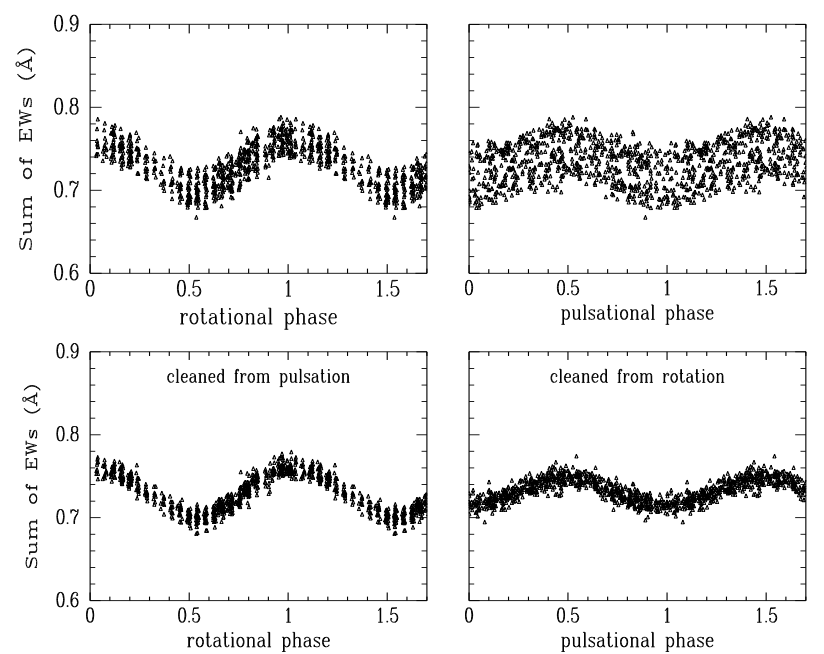

Fig. 7. Rotational and pulsational (dominant mode) variations of the sum of equivalent widths of 18 selected lines of $\mathrm{O}_{\text {II }}, \mathrm{N}_{\text {II, }}$ and $\mathrm{Si}$ III. In left (right) panels data are plotted as a function of rotational (pulsational) phase, calculated using parameters of Table 6. In the lower panels one of the two variations has been removed by subtracting a sinusoidal fit, making the remaining variation appear more clearly.

confidence in our analysis of the spectral variations corresponding to each phenomenon.

Rotational distortions of spectral lines are expected if chemical elements are not evenly distributed over the stellar surface. A large chemical spot, for example, would produce a one-wave modulation with the rotation period. This effect is discussed in Sect. 5.2. The main radial velocity variations are a consequence of the stellar pulsation (Fig. 2). In addition to the variations in the mean (disc-integrated) radial velocity, pulsational line-profile distortions are also expected according to the radial velocity distribution over the stellar disc, caused by radial and nonradial pulsations. Moreover, radial pulsations can introduce indirectly spectral variations through the variations of atmospheric parameters ( $T_{\text {eff }}$ and $\log g$ ) during the pulsational cycle (e.g. De Ridder et al. 2002). This issue is addressed in Sect. 5.3.

\subsection{Equivalent width variations}

Rotational modulation and line profile variations due to atmospheric parameter variations are clearly diagnosed through equivalent width variations (e.g. Briquet et al. 2004, 2009). To compute these differences, we selected several clean lines of various chemical elements, particularly $\mathrm{He}, \mathrm{C}, \mathrm{N}, \mathrm{O}, \mathrm{Ne}, \mathrm{Al}, \mathrm{Si}, \mathrm{S}$, and Fe. In order to avoid small systematic differences between the data sets from different instruments and in order to have the same spectral range (the same line set) we based our analysis on the CORALIE data alone. The HARPS data were only used to check for consistency. The large number of CORALIE observations and their uniform distribution in rotational and pulsational phases allows us to study one phenomenon at a time while other effects are averaged out.

A frequency analysis of the equivalent width variations of Si III lines clearly indicates a rotation period of $23.4 \mathrm{~d}$. This value can be refined using the magnetic data from Järvinen et al. (2017) spanned over years. We refit the magnetic field measurements from Järvinen et al. (2017) with a sine function obtaining $P_{\text {rot }}=$ $23.383 \pm 0.008 \mathrm{~d}$ and $\mathrm{HJD}_{|B z| \max }=2456743.5 \pm 0.9$. In the following analysis, we keep the rotational period fixed at $23.383 \mathrm{~d}$.

We measured the equivalent widths automatically for 61 spectral lines in the 646 CORALIE spectra using our own program that calculates the first five statistical moments of spectral line profiles. In order to increase the $\mathrm{S} / \mathrm{N}$, we summed the equivalent width of several lines of the same ion when possible. As expected, the equivalent widths were found to depend on both the dominant pulsation and rotation phases so that the two variations had to be fit simultaneously. As illustration, Fig. 7 shows the dominant pulsational and rotational variations for the sum of 18 selected lines of $\mathrm{O}$ II, N II, and Si III, three ions whose lines show similar behaviour. Thus, the data were fit with the sum of two sinusoidal functions. Both periods were kept fixed (values from Table 2 were adopted), and thus five free parameters were fit: the mean value $\left(W_{0}\right)$, the pulsational amplitude $\left(A_{\text {pul }}\right)$, the rotational amplitude $\left(A_{\text {rot }}\right)$, and the time of maximum for the two variations $\left(\mathrm{HJD}_{\max }(\mathrm{pul}), \mathrm{HJD}_{\max }(\mathrm{rot})\right)$.

Table 6 shows the mean behaviour for each atomic species. These results are discussed in the two following subsections.

\subsection{Rotational variations}

Most metallic lines are variable with roughly the same behaviour, while the He I lines behave opposite to metals. Figure 8 shows the average of 139 spectra around rotational phase 0.00 and the average of 129 spectra around phase 0.50 (red). The difference of the two mean spectra is plotted above the average. The opposite behaviour of metals and helium lines is clear.

Figure 9 shows for different atomic species, the sum of equivalent widths of several lines. In the lower panel we plot the magnetic field measurements from Järvinen et al. (2017). Roughly, the negative magnetic field corresponds to maximum helium and minimum metal lines. The best sinusoidal fit of the magnetic measurements would be shifted in phase by $0.09-0.10$ with respect to the chemical variation curves. However, for a proper comparison between chemical element distribution and the magnetic field geometry, a better phase coverage of the magnetic field data is needed.

Figure 10 shows in polar coordinates these variations along the rotational cycle. In this graph each symbol describes the variation of one atomic species. The radial coordinate is the mean value per line of the variation-amplitude (i.e. $A_{\text {rot }} / n$ ) and the polar coordinate the rotational phase of maximum intensity. For the origin of rotational phases we adopted the average value of times of maximum intensity of 26 lines of $\mathrm{O}_{\text {II, }} \mathrm{N}_{\text {III }}$ and $\mathrm{Si}$ III: $\operatorname{HJD}\left(\max \mathrm{O}_{\text {II, }} \mathrm{N}_{\text {II, }} \mathrm{Si}\right.$ III $)=456768.99 \pm 0.39$. This value and the adopted rotation period $(23.383 \mathrm{~d})$ are used in the calculation of rotational phases shown in Figs. 7, 9, and 10.

Helium is strengthened at phase 0.48 while all metals have a maximum at phases $\sim 0.00-0.05$. The magnetic field intensity as a function of the phase is shown in the figure in red. The continuous red line is the modulus of $B z$, while dotted lines indicate the $1 \sigma$ uncertainty interval. The rotational variation of spectral lines is presumably related to chemical spots. In this context the surface region with the strongest overabundance of helium corresponds roughly to the negative magnetic pole. We note that all magnetic measurements by Järvinen et al. (2017) are negative, so the radius vector in Fig. 10 is $-B z$.

\subsection{Pulsational variations}

In order to detect subtle spectral variations throughout the dominant pulsational cycle, we calculated average spectra in phase bins of $\Delta \phi=0.1$. The most notable differences were between the spectrum at phase 0.00 (zero radial velocity, maximum stellar 
Table 6. Parameters of the fitting of the sum of the equivalent width of several spectral lines of a given atomic species.

\begin{tabular}{|c|c|c|c|c|c|c|c|c|c|}
\hline $\begin{array}{l}\text { Ion } \\
\text { (1) }\end{array}$ & $\begin{array}{l}W_{0} \\
\mathrm{~m} \AA \\
(2)\end{array}$ & $\begin{array}{c}\sigma \\
\mathrm{mA} \\
(3)\end{array}$ & (4) & $\begin{array}{c}A_{\text {pul }} \\
\mathrm{mA} \\
(5)\end{array}$ & $\begin{array}{c}\mathrm{HJD}_{\max }(\mathrm{pul}) \\
-2456760 \\
(6)\end{array}$ & $\begin{array}{c}A_{\mathrm{pul}} / W_{0} \\
\% \\
(7)\end{array}$ & $\begin{array}{c}A_{\text {rot }} \\
\mathrm{m \AA} \\
(8)\end{array}$ & $\begin{array}{c}\mathrm{HJD}_{\max } \text { (rot) } \\
-2456760 \\
(9)\end{array}$ & $\begin{array}{c}A_{\text {rot }} / W_{0} \\
\% \\
(10)\end{array}$ \\
\hline He I & $1040.5 \pm 0.4$ & 10.72 & 3 & $1.17 \pm 0.61$ & $0.024 \pm 0.007$ & 0.1 & $13.49 \pm 0.60$ & $20.3 \pm 0.2$ & 1.3 \\
\hline $\mathrm{C}_{\text {II }}$ & $385.8 \pm 0.3$ & 6.44 & 6 & $2.17 \pm 0.37$ & $0.029 \pm 0.002$ & 0.6 & $10.82 \pm 0.36$ & $9.7 \pm 0.1$ & 2.8 \\
\hline $\mathrm{C}_{\text {III }}$ & $7.9 \pm 0.1$ & 2.02 & 1 & $0.45 \pm 0.11$ & $-0.009 \pm 0.004$ & 5.7 & $0.52 \pm 0.12$ & $12.7 \pm 0.8$ & 6.6 \\
\hline $\mathrm{N}_{\text {II }}$ & $374.1 \pm 0.2$ & 5.91 & 12 & $6.14 \pm 0.34$ & $0.069 \pm 0.001$ & 1.6 & $16.90 \pm 0.33$ & $9.1 \pm 0.1$ & 4.5 \\
\hline $\mathrm{O}_{\text {II }}$ & $337.5 \pm 0.2$ & 5.02 & 10 & $9.99 \pm 0.29$ & $0.070 \pm 0.000$ & 3.0 & $14.85 \pm 0.28$ & $8.8 \pm 0.1$ & 4.4 \\
\hline $\mathrm{Ne}$ I & $32.9 \pm 0.1$ & 2.60 & 1 & $0.38 \pm 0.15$ & $0.025 \pm 0.006$ & 1.2 & $0.73 \pm 0.15$ & $10.4 \pm 0.8$ & 2.2 \\
\hline $\mathrm{Mg}_{\mathrm{II}}$ & $101.6 \pm 0.1$ & 1.88 & 1 & $1.46 \pm 0.11$ & $0.021 \pm 0.001$ & 1.4 & $2.85 \pm 0.11$ & $10.0 \pm 0.1$ & 2.8 \\
\hline Al III & $100.6 \pm 0.1$ & 3.48 & 4 & $1.14 \pm 0.20$ & $0.067 \pm 0.002$ & 1.1 & $5.53 \pm 0.20$ & $10.0 \pm 0.1$ & 5.5 \\
\hline Si II & $90.0 \pm 0.2$ & 4.21 & 6 & $3.56 \pm 0.24$ & $0.025 \pm 0.001$ & 4.0 & $4.23 \pm 0.24$ & $9.6 \pm 0.2$ & 4.7 \\
\hline Si III & $244.8 \pm 0.1$ & 3.48 & 4 & $4.59 \pm 0.20$ & $0.069 \pm 0.001$ & 1.9 & $7.98 \pm 0.20$ & $9.2 \pm 0.1$ & 3.3 \\
\hline Si IV & $13.6 \pm 0.1$ & 2.45 & 2 & $0.76 \pm 0.14$ & $0.070 \pm 0.003$ & 5.6 & $0.78 \pm 0.14$ & $9.2 \pm 0.7$ & 5.8 \\
\hline $\mathrm{S}_{\text {II }}$ & $49.9 \pm 0.1$ & 3.14 & 4 & $1.19 \pm 0.18$ & $0.026 \pm 0.002$ & 2.4 & $2.08 \pm 0.18$ & $10.3 \pm 0.3$ & 4.2 \\
\hline $\mathrm{S}_{\text {III }}$ & $39.1 \pm 0.1$ & 2.85 & 2 & $0.77 \pm 0.16$ & $0.066 \pm 0.003$ & 2.0 & $1.66 \pm 0.16$ & $8.0 \pm 0.4$ & 4.3 \\
\hline $\mathrm{Fe}_{\text {II }}$ & $6.6 \pm 0.1$ & 2.02 & 1 & $0.30 \pm 0.11$ & $0.034 \pm 0.006$ & 4.6 & $0.04 \pm 0.12$ & $13.6 \pm 10.6$ & 0.6 \\
\hline $\mathrm{Fe}_{\text {III }}$ & $82.8 \pm 0.1$ & 3.63 & 4 & $1.03 \pm 0.21$ & $0.068 \pm 0.003$ & 1.2 & $3.19 \pm 0.21$ & $10.4 \pm 0.2$ & 3.9 \\
\hline
\end{tabular}

Notes. Columns are (1) ion; (2) mean value; (3) standard deviation of residuals; (4) number of lines; (5) amplitude of the pulsational variations; (6) time of maximum line intensity for pulsational variations; (7) pulsational variation amplitude as a percent of the line intensity; $(8,9$, and 10$)$ same as 5,6 , and 7 , but for rotational variations.

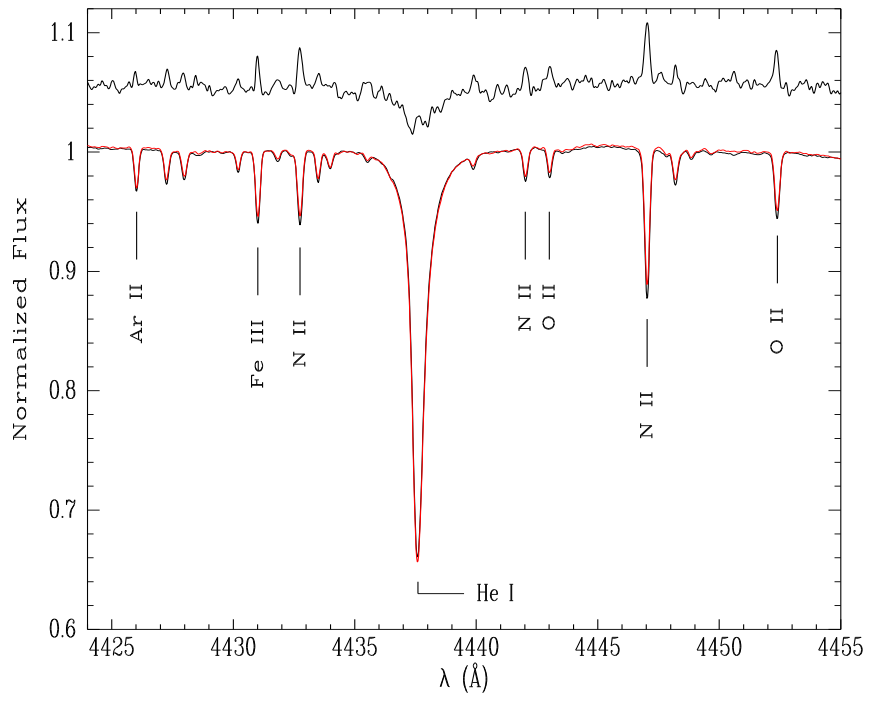

Fig. 8. Spectral variations related to the stellar rotation. Mean spectra at rotational phases 0.00 (black) and 0.50 (red) are shown in a small spectral window containing various helium and metal lines. In the upper part the difference between the two spectra is shown (expanded by a factor 5 and shifted +1.05$)$.

radius) and at phase 0.50 (zero radial velocity, minimum stellar radius). In Fig. 11 we compare for several diagnostic lines the spectrum at maximum radius (average of 54 spectra with pulsational phase in the range $0.95-0.05)$ and at minimum radius (average of 67 spectra in the range $0.45-0.55$ ). In all the elements for which there are spectral lines corresponding to two or three different ionization states, the lines of higher ionization state are stronger near phase 0.50 , which corresponds to minimum stellar radius. More specifically, at phase 0.00 , the lines of $\mathrm{He}_{\mathrm{I}}, \mathrm{Ne}$ I, $\mathrm{Mg}$ II, Si II, and Fe II are stronger, while at phase 0.50 the lines of $\mathrm{C}_{\text {IIII, }} \mathrm{N}_{\text {II }}, \mathrm{O}_{\text {II, }}$ and $\mathrm{Si}$ III-IV are stronger.

The same can be seen in Fig. 12, whose format is similar to Fig. 10. In this case the distance to the origin represents the amplitude of the pulsational variation as a percent of the line intensity. The population of atoms in low ionization states is maximum near phase 0.0 and that of highly ionized atoms is maximum at phase 0.5. Two low-quality points (C III and Fe II) have been omitted. This analysis suggests that $T_{\text {eff }}$ is higher at phase 0.50 .

In order to evaluate quantitatively the temperature difference between phases 0.0 and 0.5 , we proceeded as follows. To derive the dependence of the intensity of selected spectral lines with temperature, we calculated a modified synthetic spectrum of HD 96446 with a temperature $300 \mathrm{~K}$ hotter. Then we measured the relative difference in the equivalent width of several strong unblended metallic lines. If we assume that in this small temperature range the relative intensity of spectral lines varies linearly with temperature, then the same line-variation pattern is expected to be observed in HD 96446 between phases 0.0 and 0.5 , up to a multiplicative factor. This scale factor is smaller (larger) than one if the temperature difference between phases 0.0 and 0.5 is lower (higher) than $300 \mathrm{~K}$. We therefore calculated in the same way the line intensity differences between the average spectrum of HD 96446 at phase 0.0 and that at phase 0.5 .

In Fig. 13 we plot the pulsational variations of line equivalent widths observed in HD 96446 as a function of the equivalent width differences calculated for $\Delta T_{\text {eff }}=300 \mathrm{~K}$. Lines of the same ion are plotted with the same symbol. Positive (negative) values correspond to lines whose intensity increase (decrease) with temperature. The slope of point distribution in Fig. 13 gives the observed temperature difference in units of $300 \mathrm{~K}$. From a linear regression of the data we obtained a value of $\Delta T_{\text {eff }}=99 \pm 4 \mathrm{~K}$ for the amplitude of pulsational variations in the temperature. We neglected in these calculations the spectral variations due to variations in surface gravity. In fact, the variation in $\log g$ is expected to be very small. From the pulsational variations of the radius $\left(\Delta R=15-20 \times 10^{3} \mathrm{~km}\right.$, see Sect. 7) and the estimated radius $\left(6.1 R_{\odot}\right.$, see Table 5$)$, we estimate the variation of gravity to be $\Delta \log g=0.003$, which has no noticeable effect on the spectral morphology. 


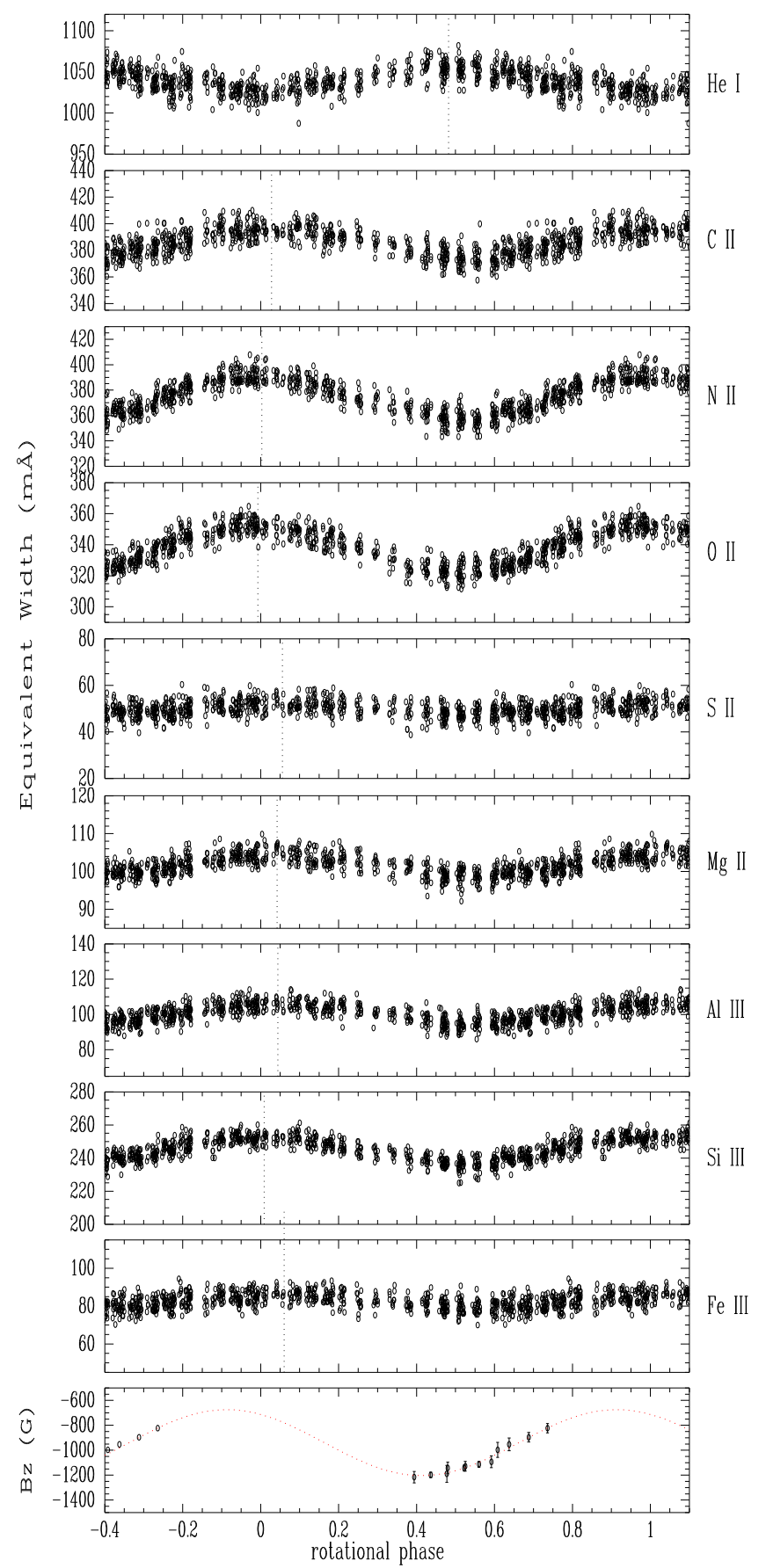

Fig. 9. Rotational variation of spectral lines. Each panel shows the sum of equivalent widths of several spectral lines of the same ion, as detailed in Table 6 . Phases of maximum intensity are marked with vertical dotted lines. The lower panel shows the longitudinal magnetic field.

\section{Mode identification}

When studying B-type pulsators, a frequency analysis on the statistical moments must be complemented by a pixel-by-pixel frequency analysis across the line profile since a 2D analysis is more sensitive to the detection of high-degree modes than a 1D analysis on integrated quantities. Thus, it can lead to the detection of additional independent pulsation frequencies (e.g. Telting et al. 1997; Briquet et al. 2005). Therefore, we performed a $2 \mathrm{D}$ frequency search by means of the tools available

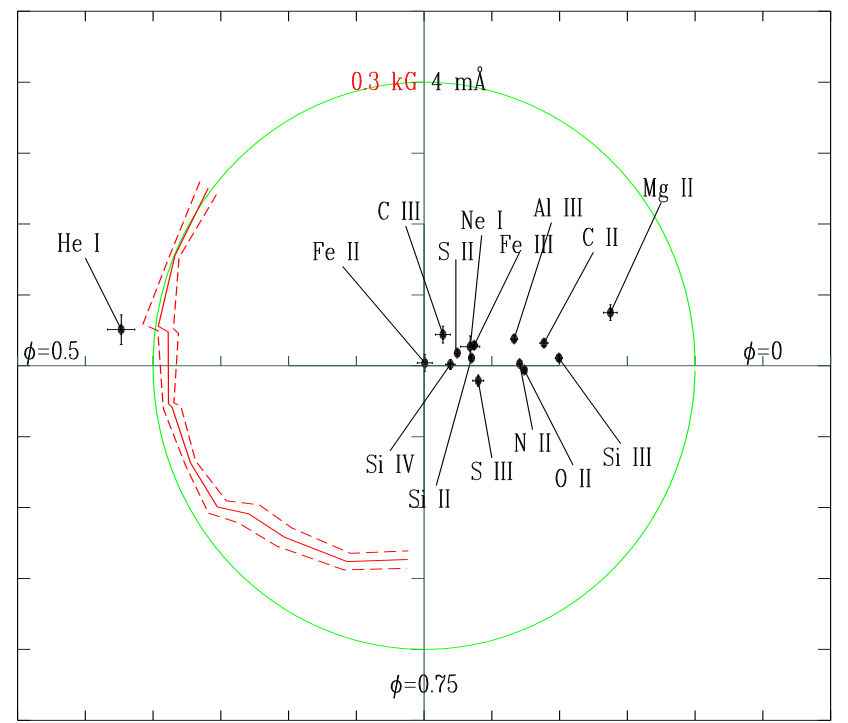

Fig. 10. Spectral variations related to the stellar rotation. Polar coordinates are used: the radius vector of each point represents the amplitude of the variations in the equivalent width (in $m \AA$ ) and the polar angle is the rotational phase of maximum intensity. The modulus of the longitudinal magnetic field, as a function of phase, is shown by the red line (red dashed lines show $\pm 1 \sigma$ interval). The scale of the radial coordinate is given by the green circle, which corresponds to $4 \mathrm{~m} \AA$ (line intensity) and $0.3 \mathrm{kG}$ (magnetic field).

in the software package FAMIAS ${ }^{6}$ (Zima 2008). Our analysis was applied on the processed CORALIE spectra after removing the orbital motion and using the heliocentric Julian dates corrected by the light-time effect. In Fig. 14 the mean of all Fourier spectra across the Si III $4552 \AA$ line is shown for subsequent steps of pre-whitening. The significance of a frequency cannot be derived from the average Fourier spectrum; the procedure is as follows. The wavelength at which the given frequency has the highest amplitude is determined. Then, the Fourier spectrum at this pixel is computed and the frequency is retained if its amplitude exceeds 4 times the S/N (Breger et al. 1993). The noise level is calculated in a $5 \mathrm{~d}^{-1}$ interval centred on the frequency of interest. We determined successively: $f_{1}, 2 f_{1}, f_{\text {rot }}, 3 f_{1}$, $f_{2}, f_{1}+f_{2}, f_{1}+f_{\text {rot }}$, and $f_{1}-f_{2}$. The frequencies detected in the equivalent widths and radial velocities are clearly seen, but no additional frequency is detected.

Neiner et al. (2012a) discovered the $\beta$ Cep nature of HD 96446 and suggested the dominant pulsation to be a radial mode but no empirical mode identification was achieved. Two independent methods based on spectroscopy are usually used to identify the modes of $\beta$ Cep stars, namely the moment method (Briquet \& Aerts 2003) and the Fourier parameter fit (FPF) method (Zima 2006), which are implemented in FAMIAs. The moment method, based on the first two statistical moments, is better suited to low-degree pulsation modes; instead, there is no upper limit for the degree $\ell$ for the FPF method. For the latter, however, the mode identification might be impossible for a very small value of $v \sin i$, as is the case for HD 96446, while this constraint does not apply to the moment method. As this issue is more critical for a large pulsation velocity relative to the projected rotational velocity, we attempted to apply both methods to the target.

6 FAMIAS has been developed in the framework of the FP6 European Coordination Action HELAS. 

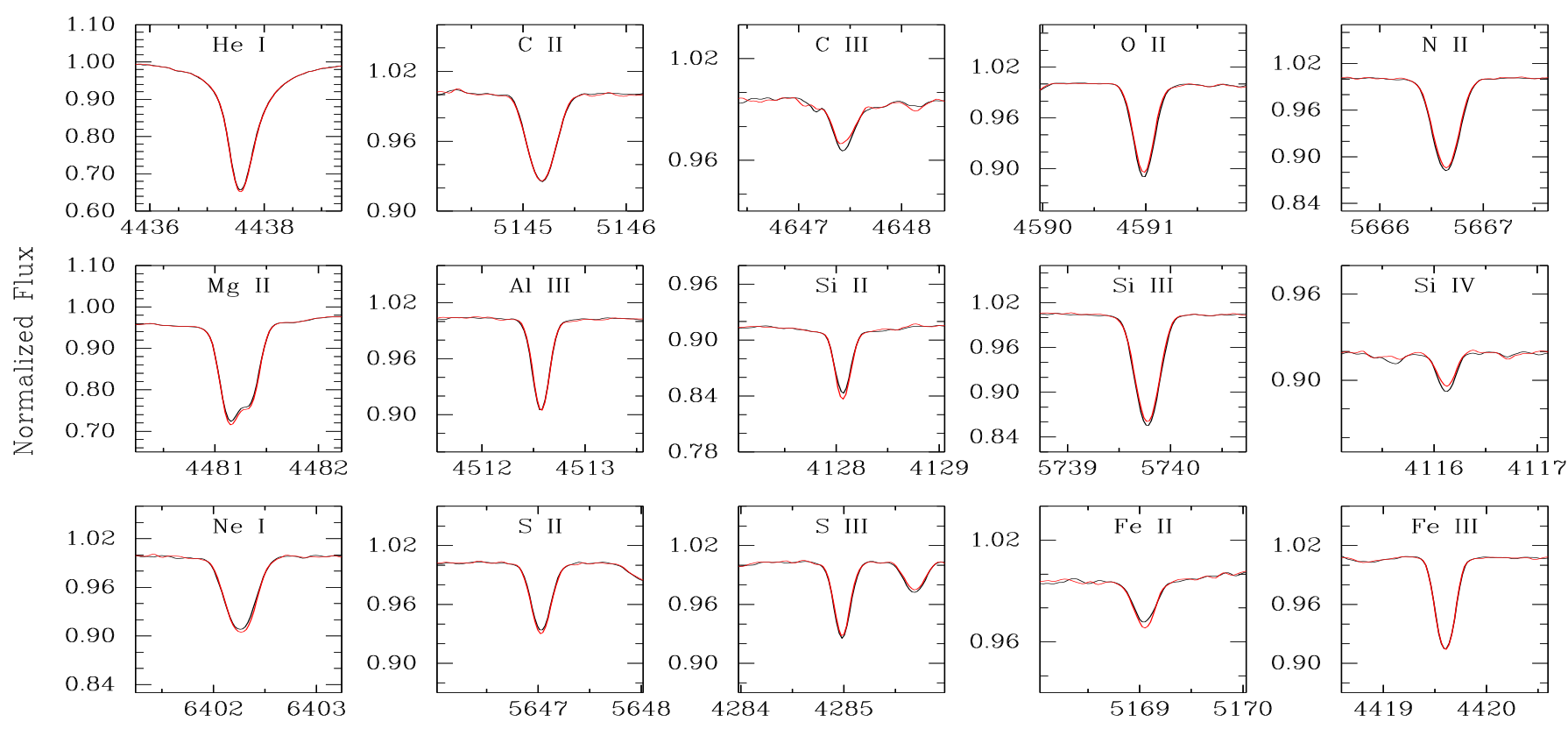

Wavelength $(\AA)$

Fig. 11. Spectral variations related with the dominant stellar pulsation: comparison between the mean spectrum at phase 0.50 (maximum radius, black line) and 0.00 (minimum radius, red line) for several lines.

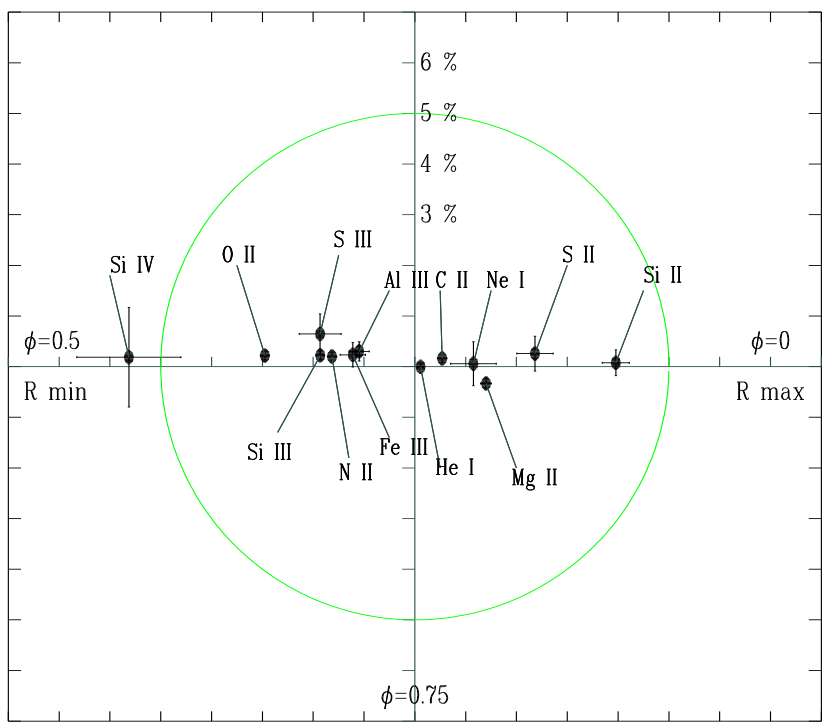

Fig. 12. Spectral variations related with the dominant stellar pulsation in polar coordinates. The radius vector of each point represents the amplitude of the variations as a percent of the mean line intensity; the polar angle is the rotational phase of maximum intensity. The green circle corresponds to a line variation of $5 \%$.

We refer to Desmet et al. (2009) for a detailed explanation of a typical application of these techniques to $\beta$ Cep stars. Figure 15 shows that the amplitude and phase behaviour across the line profile for the dominant mode is typical of a radial mode, which cannot be distinguished from a dipole $(\ell, m)=(1,0)$ mode with a small inclination (Aerts et al. 2010). For the second mode, we have $m>0$ (where a positive $m$-value denotes a prograde mode) in order to fit the phase across the profile. Moreover, from a visual inspection of the solutions, the matching between the observed and synthetic amplitudes and phases across the profile is the most satisfactory for $(\ell, m)=(2,2)$ or $(\ell, m)=(3,2)$ or $(\ell, m)=(3,3)$ for an inclination angle compatible with the

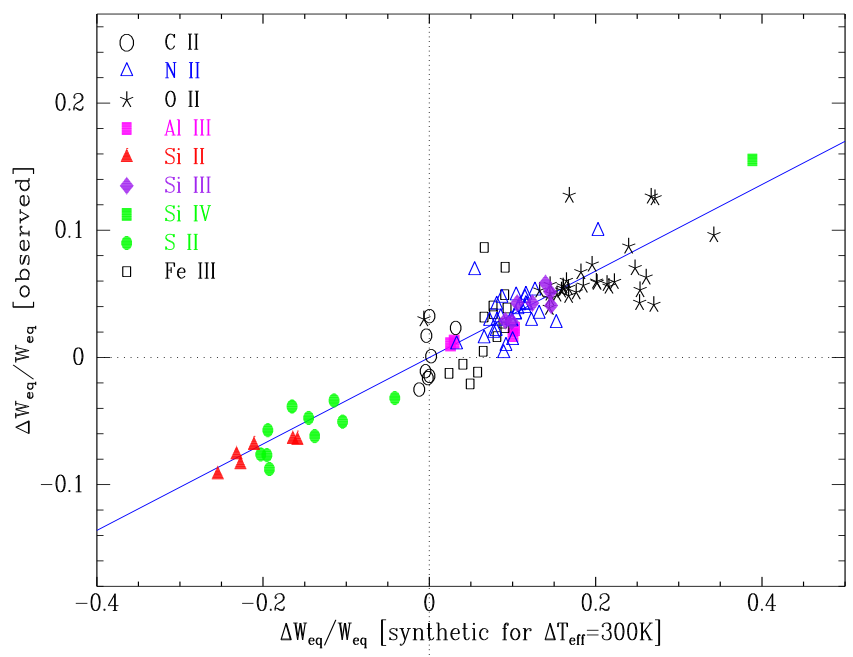

Fig. 13. Comparison of the relative differences between the observed equivalent widths and those calculated using synthetic spectra. Spectral lines of the same ion are plotted with the same symbol. The slope of the least-squares linear fit (continuous line) represents the temperature variation along the pulsational cycle in units of $300 \mathrm{~K}$.

constraints obtained from the magnetic model (i.e. $i=18 \pm 15^{\circ}$ ). The moment method gives fully compatible results, excluding the $(\ell, m)=(3,3)$ solution. In Fig. 15, we show the $(\ell, m)=(2,2)$ solution.

\section{Main pulsational mode}

In the previous section we identify the main pulsational mode as a radial $(0,0)$ or a dipole $(1,0)$ mode under low inclination. In this section we analyse the variation in physical parameters of this star assuming it is pulsating radially. From these calculations we conclude that the most probable configuration is the low-inclination dipole. 


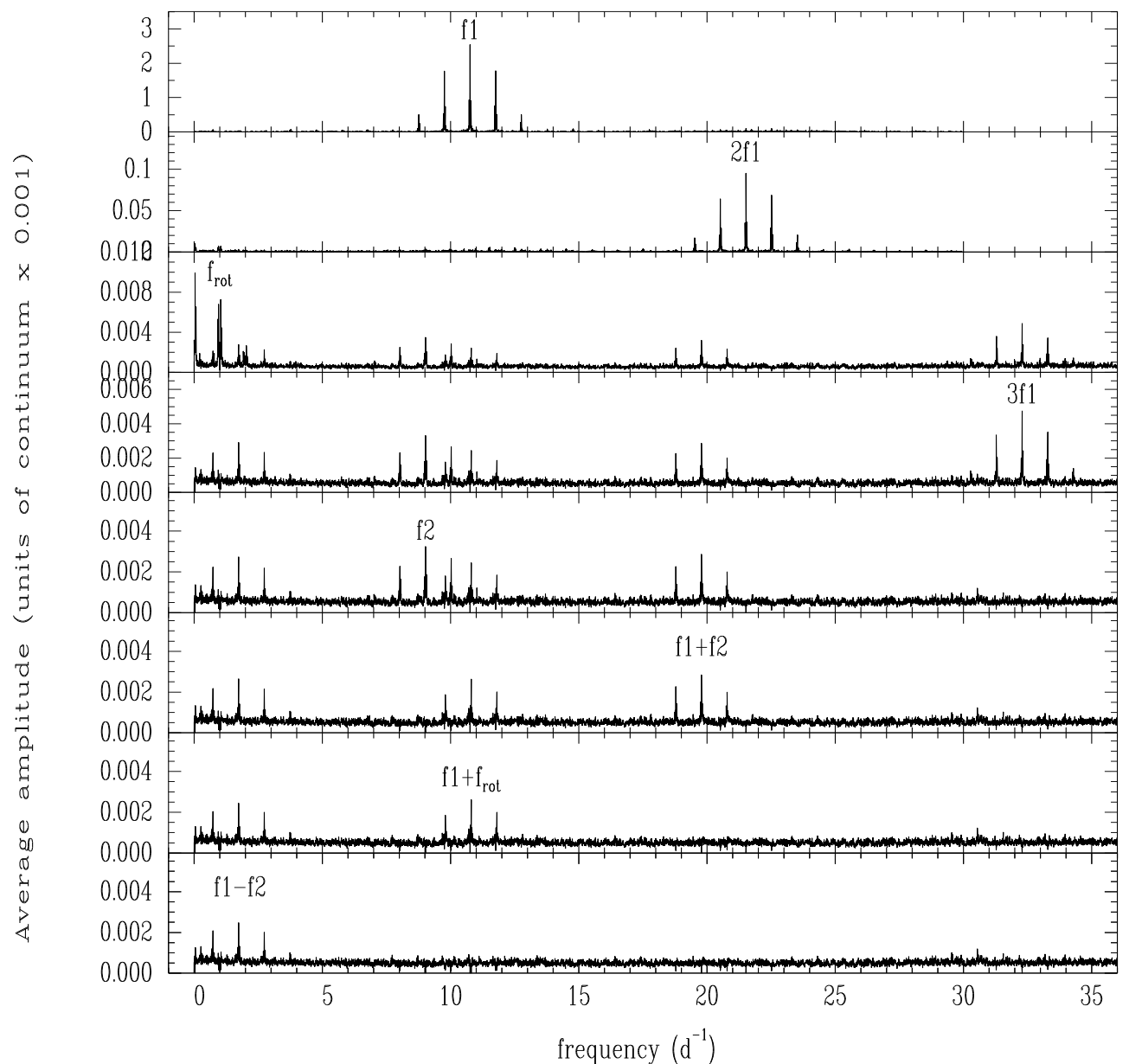

Fig. 14. Amplitude periodogram corresponding to the average of all Fourier spectra across the Si III $4552 \AA$ profile. The different panels show successive stages of pre-whitening (top to bottom).
Assuming that the main pulsation is a radial mode, it is possible to derive the variation in the stellar radius in absolute units by integration of the radial velocity curve. For a spherical star the relation between the radius time-derivative and the radial velocity is $\dot{R} \approx-p \mathrm{RV}$, where the projection factor is $p=1.433 \pm 0.005$ for a stellar disc with linear limb-darkening law with coefficient $0.28-0.35$. This factor was calculated by direct integration of a limb-darkened stellar disc, but it is also consistent with other works in early-type stars (Nardetto et al. 2013).

In the case of HD 96446 the total radius variation in a pulsational cycle is

$$
\Delta R=\frac{p}{\pi} \cdot P_{1} \cdot A_{1}=15500 \pm 60 \mathrm{~km} \text {. }
$$

According to the radial velocity analysis, the time of maximum radius is $\mathrm{HJD}=2456760.0244$ (corresponding to $\phi_{1}=0$ ). The time of maximum equivalent width of highly ionized metals (weighted average of $\mathrm{N}_{\text {II, }}$ O II, Si III-IV, S III, and Fe III lines) due to pulsation is $\mathrm{HJD}=2456760.0694\left(\phi_{1}=0.49\right)$, corresponding approximately to the minimum stellar radius. Thus, the temperature is higher when the stellar radius is minimal.

Flux variations related to the radius and temperature variations might also be expected. We confront the expected variations with the light curve calculated using the HipParcos photometry. This light variation is related to the radius and temperature variations through

$\frac{\Delta I}{I}=2 \cdot \frac{\Delta R}{R}+\frac{\Delta F}{F}=2 \cdot \frac{\Delta R}{R}+1.85 \cdot \frac{\Delta T_{\mathrm{eff}}}{T_{\mathrm{eff}}}$,

where the surface flux density $F$ has been expressed as a power law of the effective temperature, with a coefficient estimated from synthetic spectra in this temperature range. Using the estimated values for the radius and temperature (Sect. 4), and the temperature variation (Sect. 5.3), we calculate that the light variations would be insignificant: $\frac{\Delta I}{I}=0.001 \pm 0.001$.

The Hipparcos photometry, however, shows clearly the main pulsational mode (Fig. 16). The fitting of the curve with a sinusoidal function gives a semi-amplitude of $5.9 \pm 0.9 \mathrm{mmag}$. The total observed flux variation is then $\frac{\Delta I}{I}=0.011 \pm 0.002$. The maximum brightness is at $\mathrm{HJD}=2448290.060$ phase $0.53 \pm 0.02$, close to the phase of minimum radius. This light variation is only compatible with the observed radius variation if the temperature variation is $220 \pm 30 \mathrm{~K}$, which is not consistent with the observed spectral variations (Sect. 5.3).

On the other hand, in the case of a dipolar mode observed under a low-inclination angle, the nodal line is close to the limb of the stellar disc so there is no appreciable variation in the area of apparent disc. The light variations would correspond in this case only to temperature variations. Accurate calculations are not possible since they required assuming a fixed and known value of inclination, in addition to modelling the temperature and flux distribution. However, we note that a temperature variation of the order of $100 \mathrm{~K}$ and $\Delta R \sim 0$ are consistent with a flux variation of the order of $0.01 \mathrm{mag}$, as observed.

\section{Summary and conclusions}

HD 96446 is a very notable binary star, whose primary is an early-type star that is magnetic and chemically peculiar with chemical spots, and that pulsates in at least two different modes. We report that HD 96446 is a spectroscopic binary with an 

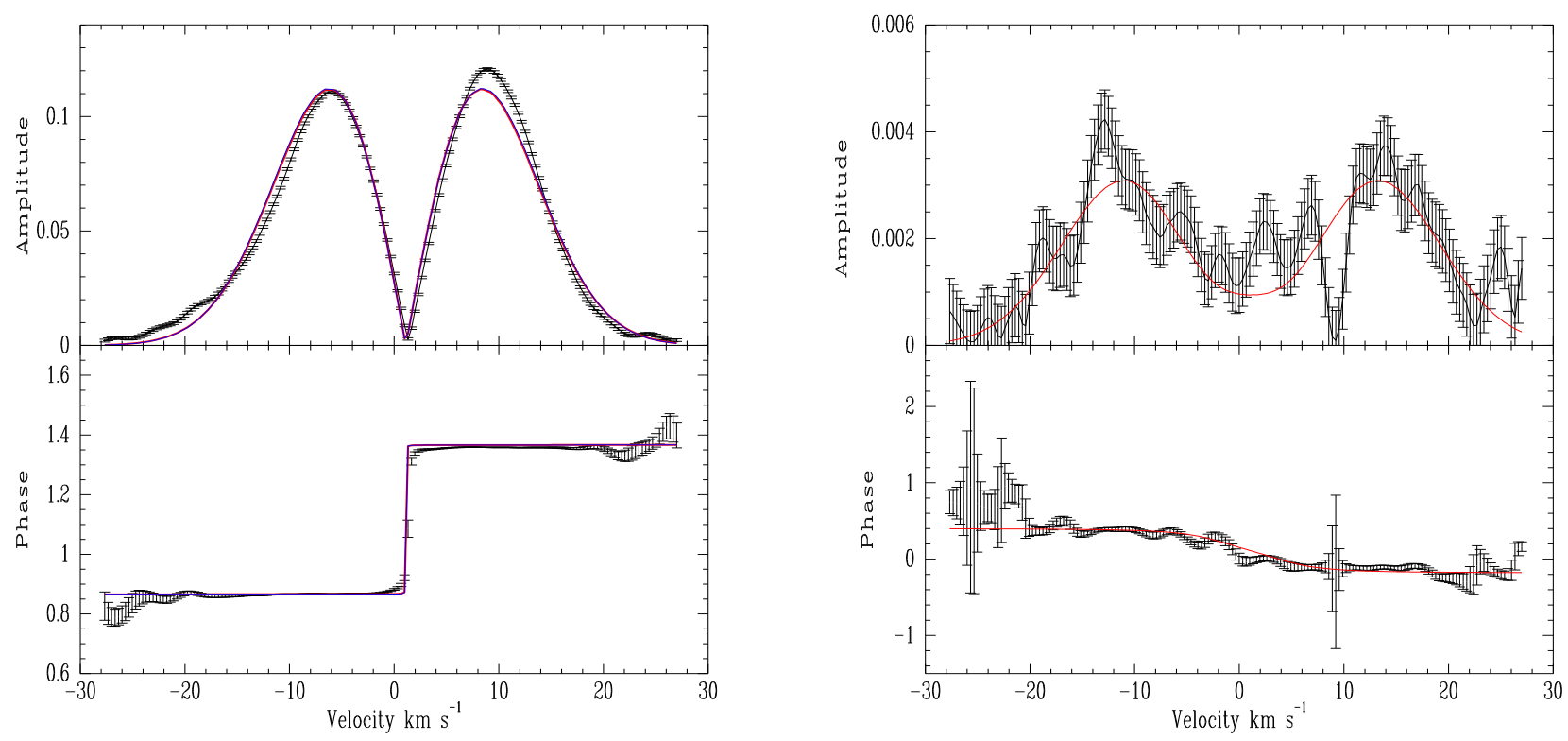

Fig. 15. Amplitude and phase distributions of the frequencies $f_{1}$ (left) and $f_{2}$ (right) across the Si III $4552 \AA$ profile. Points with error bars are observations, while full lines are theoretical distributions. In the left panel, models for $(l, m)=(0,0)$ (red line) and pole-on $(l, m)=(1,0)$ (blue line), which are indistinguishable at the plotting scale, are shown. In the right panel the theoretical curve corresponds to $(l, m)=(2,2)$. The amplitude is expressed in units of continuum and the phase in $2 \pi$ radians.

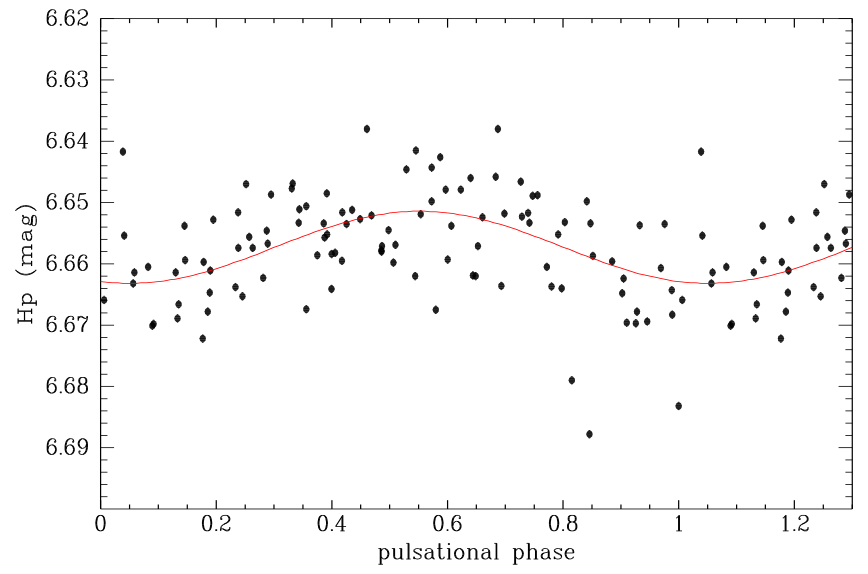

Fig. 16. HiPPARCos photometry phased with the main pulsation period.

eccentric ( $e=0.59$ ) orbit with a semi-axis $a \approx 3.8 \mathrm{AU}$. The primary is a helium strong B2IV-type star and the secondary has a spectral type $\sim \mathrm{A} 0$. The mass-ratio is relatively low, $q=0.29$, resulting in a visual light-ratio of the components of only $\sim 0.05$. The primary star has a magnetic field that can be described as a dipole of $4.6 \mathrm{kG}$ slightly inclined with respect to the rotational axis. The star rotates with a period of 23.4 days. The rotation axis has very low inclination, such that the positive magnetic pole is always hidden to the observer.

The surface chemical composition is abnormal, with a very high abundance of helium, 0.6 by number of atoms, which represent $85 \%$ of the photospheric mass. Chemical elements are not evenly distributed over the stellar surface. Helium is concentrated on the visible magnetic pole (the negative pole), while metals are more abundant at intermediate latitudes. Even though the abundance analysis has been performed on a mean spectrum calculated by averaging all rotational phases, the mean surface abundances may differ significantly from the values calculated here, due to the particular geometric configuration of the system. In fact, the low inclination of the rotational axis implies that a part of the stellar surface is permanently hidden to the observer, including the positive magnetic pole. This configuration prevents us from estimating mean surface abundances or discussing the chemical surface distribution in more detail.

This star is a pulsating star with at least two pulsational modes with periods of 0.09292 and 0.1108 days. The main mode is probably a low-inclination, dipole mode $(l, m)=(1,0)$. The second pulsation mode corresponds to $(l, m)=(2,2)$ or to a poleon $(l, m)=(3,2)$ configuration. Temperature variations over the cycle were detected in the spectrum; the highest temperature corresponds approximately to the minimum radius. Given that HD 96446 is a pulsator with at least two modes and that the rotational period is now precisely known, it is a very promising target for an asteroseismic modelling.

Since the pulsational frequency is proportional to the square root of the mean stellar density, pulsation is a sensitive indicator of the stellar evolution (see e.g. Neilson \& Ignace 2015). For a $9 M_{\odot}$ star, the expected period variation is $\dot{P} / P \sim 7 \times 10^{-8} \mathrm{yr}^{-1}$. In the present work the main pulsational period was determined with a relative error $\Delta P / P \sim 10^{-7}$. Thus, if a similar study is repeated for this object a few years from now, it will be possible to measure the stellar expansion rate during the main-sequence evolution.

Acknowledgements. M.F.N. acknowledges the support of the Austrian Science Fund (FWF) via a Lise-Meitner Fellowship under project number N-1868-NBL. Some of the data presented in this paper were obtained from the Mikulski Archive for Space Telescopes (MAST). STScI is operated by the Association of Universities for Research in Astronomy, Inc., under NASA contract NAS526555. Support for MAST for non-HST data is provided by the NASA Office of Space Science via grant NNX09AF08G and by other grants and contracts. A.T. is a Research Associate at the Belgian Scientific Research Fund (F.R.S-FNRS).

\section{References}

Aerts, C., Christensen-Dalsgaard, J., \& Kurtz, D. W. 2010, Asteroseismology (Berlin, Heidelberg: Springer)

Baranne, A., Queloz, D., Mayor, M., et al. 1996, A\&AS, 119, 373

Becker, S. R. 1998, ASP Conf. Ser., 131, 137 
Becker, S. R., \& Butler, K. 1988, A\&A, 201, 232

Belkacem, K., Samadi, R., Goupil, M.-J., et al. 2009, Science, 324, 1540

Bertaux, J. L., Lallement, R., Ferron, S., Boonne, C., \& Bodichon, R. 2014, A\&A, 564, A46

Bohlender, D. A., Landstreet, J. D., Brown, D. N., \& Thompson, I. B. 1987, ApJ, 323,325

Borra, E. F., \& Landstreet, J. D. 1979, ApJ, 228, 809

Borra, E. F., Landstreet, J. D., \& Mestel, L. 1982, ARA\&A, 20, 191

Breger, M., Stich, J., Garrido, R., et al. 1993, A\&A, 271, 482

Briquet, M., \& Aerts, C. 2003, A\&A, 398, 687

Briquet, M., Aerts, C., Lüftinger, T., et al. 2004, A\&A, 413, 273

Briquet, M., Lefever, K., Uytterhoeven, K., \& Aerts, C. 2005, MNRAS, 362, 619

Briquet, M., Uytterhoeven, K., Morel, T., et al. 2009, A\&A, 506, 269

Briquet, M., Neiner, C., Aerts, C., et al. 2012, MNRAS, 427, 483

Butler, K., \& Giddings, J. R. 1985, Newsletter of Analysis of Astronomical Spectra, 9 (London: Univ. London)

Buysschaert, B., Aerts, C., Bowman, D. M., et al. 2018, A\&A, 616, A148

Castro, N., Fossati, L., Hubrig, S., et al. 2017, A\&A, 597, L6

Cutri, R. M., Skrutskie, M. F., van Dyk, S., et al. 2003, VizieR Online Data Catalog: II/246

De Ridder, J., Dupret, M.-A., Neuforge, C., \& Aerts, C. 2002, A\&A, 385, 572

Desmet, M., Briquet, M., Thoul, A., et al. 2009, MNRAS, 396, 1460

Dziembowski, W. A., \& Pamiatnykh, A. A. 1993, MNRAS, 262, 204

Ekström, S., Georgy, C., Eggenberger, P., et al. 2012, A\&A, 537, A146

Ferrario, L., Pringle, J. E., Tout, C. A., \& Wickramasinghe, D. T. 2009, MNRAS, 400, L71

Gaia Collaboration (Brown, A. G. A., et al.) 2018, A\&A, 616, A1

Giddings, J. R. 1981, PhD Thesis, Univ. London

González, J. F., Hubrig, S., Przybilla, N., et al. 2017, MNRAS, 467, 437

Heber, U., Moehler, S., Napiwotzki, R., Thejll, P., \& Green, E. M. 2002, A\&A, 383,938

Henrichs, H. F., de Jong, J. A., Donati, D.-F., et al. 2000, ASP Conf. Ser., 214, 57

Hirsch, H. A. 2009, PhD Thesis, Univ. Erlangen-Nürnberg

Hubrig, S., Briquet, M., Schöller, M., et al. 2006, MNRAS, 369, L61

Hunger, K., \& Groote, D. 1999, A\&A, 351, 554

Järvinen, S. P., Hubrig, S., Ilyin, I., Schöller, M., \& Briquet, M. 2017, MNRAS, 464, L85

Krtička, J., \& Kubát, J. 2001, A\&A, 369, 222

Kupfer, T., Przybilla, N., Heber, U., et al. 2017, MNRAS, 471, 877

Kurucz, R. 1993, CD-ROM No. 13 (Cambridge, Mass.: SAO)

Kurucz, R. L. 1996, ASP Conf. Ser., 108, 160

Mathis, S., \& Zahn, J.-P. 2005, A\&A, 440, 653
Mathys, G. 1991, A\&AS, 89, 121

Mermilliod, J. C. 1997, VizieR Online Data Catalog: II/168

Michaud, G., Charland, Y., \& Megessier, C. 1981, A\&A, 103, 244

Morel, T., \& Butler, K. 2008, A\&A, 487, 307

Morel, T., Butler, K., Aerts, C., Neiner, C., \& Briquet, M. 2006, A\&A, 457, 651

Moss, D. 2003, A\&A, 403, 693

Nardetto, N., Mathias, P., Fokin, A., et al. 2013, A\&A, 553, A112

Neilson, H. R., \& Ignace, R. 2015, A\&A, 584, A58

Neiner, C., Henrichs, H. F., Floquet, M., et al. 2003, A\&A, 411, 565

Neiner, C., Landstreet, J. D., Alecian, E., et al. 2012a, A\&A, 546, A44

Neiner, C., Alecian, E., Briquet, M., et al. 2012b, A\&A, 537, A148

Nelder, J. A., \& Mead, R. 1965, Comput. J., 7, 308

Nieva, M. F., \& Przybilla, N. 2006, ApJ, 639, L39

Nieva, M. F., \& Przybilla, N. 2007, A\&A, 467, 295

Nieva, M. F., \& Przybilla, N. 2008, A\&A, 481, 199

Nieva, M.-F., \& Przybilla, N. 2012, A\&A, 539, A143

Nieva, M.-F., \& Przybilla, N. 2014, A\&A, 566, A7

Przybilla, N. 2005, A\&A, 443, 293

Przybilla, N., \& Butler, K. 2001, A\&A, 379, 955

Przybilla, N., \& Butler, K. 2004, ApJ, 609, 1181

Przybilla, N., Butler, K., Becker, S. R., Kudritzki, R. P., \& Venn, K. A. 2000, A\&A, 359, 1085

Przybilla, N., Butler, K., Becker, S. R., \& Kudritzki, R. P. 2001, A\&A, 369, 1009 Przybilla, N., Butler, K., Heber, U., \& Jeffery, C. S. 2005, A\&A, 443, L25

Przybilla, N., Nieva, M. F., Irrgang, A., \& Butler, K. 2013, EAS Publ. Ser., 63, 13

Przybilla, N., Fossati, L., Hubrig, S., et al. 2016, A\&A, 587, A7

Queloz, D., Mayor, M., Weber, L., et al. 2000, A\&A, 354, 99

Rybicki, G. B., \& Hummer, D. G. 1991, A\&A, 245, 171

Smith, K. C. 1996, Ap\&SS, 237, 77

Telting, J. H., Aerts, C., \& Mathias, P. 1997, A\&A, 322, 493

Tutukov, A. V., \& Fedorova, A. V. 2010, Astron. Rep., 54, 156

van Leeuwen, F. 2007, in Hipparcos, the New Reduction of the Raw Data (Berlin, Heidelberg: Springer)

Vrancken, M., Butler, K., \& Becker, S. R. 1996, A\&A, 311, 661

Wickramasinghe, D. T., Tout, C. A., \& Ferrario, L. 2014, MNRAS, 437, 675

Zahn, J.-P. 2011, IAU Symp. Ser., 272, 14

Zboril, M., \& North, P. 2000, Contrib. Astron. Obs. Skalnate Pleso, 30, 12

Zboril, M., North, P., Glagolevskij, Y. V., \& Betrix, F. 1997, A\&A, 324, 949

Zima, W. 2006, A\&A, 455, 227

Zima, W. 2008, Commun. Asteroseismol., 155, 17 